\title{
TITTLE: Cardioprotective Effect of New NIL10 Nanoparticles Through IL10 Receptor/STAT3 Activation in Mice and Pigs Subjected Acute Myocardial Infarction
}

\author{
Laura Tesoro \\ Universidad Francisco de Vitoria \\ Laura Botana \\ Universidad Francisco de Vitoria \\ Rafael Ramírez-Carracedo \\ Universidad Francisco de Vitoria \\ Ignacio Hernández
}

Universidad Francisco de Vitoria

Javier Díez-Mata

Universidad Francisco de Vitoria

Nunzio Alcharani

Universidad Francisco de Vitoria

Marta Saura

Universidad de Alcala

Beatriz Jiménez-Guirado

Universidad Francisco de Vitoria

Karina Ovejero-Paredes

Universidad Complutense de Madrid

Marco Filice

Universidad Complutense de Madrid

Jose Luis Zamorano

Hospital Universitario Ramon y Cajal

Carlos Zaragoza ( $\nabla$ c.zaragoza.prof@ufv.es )

Universidad Francisco de Vitoria https://orcid.org/0000-0002-1706-8592

\section{Research Article}

Keywords: Acute myocardial infarction, Interleukin-10, nanoparticles, STAT-3, NF-kB

Posted Date: January 19th, 2022 
DOI: https://doi.org/10.21203/rs.3.rs-1228158/v1

License: (c) (i) This work is licensed under a Creative Commons Attribution 4.0 International License. Read Full License 


\section{Abstract}

Rationale: Early response after acute myocardial infarction (AMI) is crucial to prevent extensive cardiac necrosis. Interleukin-10 (IL-10) is a key antiinflammatory cytokine, whose expression is closely associated with macrophage polarization towards inflammation resolution.

Results: We synthesized NIL10, a micelle-based nanoparticle conjugated to IT9302, a peptide homologue to IL-10 functional domain, with the aim of targeting IL-10 receptor in mice and pigs subjected to AMI. Intravenous administration of $1 \mathrm{mg} / \mathrm{kg} \mathrm{NIL} 10$ improved the cardiac function of mice and pigs subjected to $\mathrm{AMI}$, as shown by a significant recovery of the left ventricle ejection fraction (LVEF) by days 3 and 7 after AMI, when compared to the levels found in animals injected with NIL10SC, a nanoparticle conjugated with the peptide in a scrambled orientation, in which inflammatory foci and fibrosis were strongly elevated. In IL-10 null mice subjected to AMI, NIL10 also improved heart contractility, while in IL10 receptor deficient animals, NIL10 had no effect. To test whether NIL10 may induce macrophage polarization, M2 macrophage populations were increased after day 3 of reperfusion, in which antiinflammatory cytokines, including IL4, IL7, IL10, IL13, IL16 and IL27 were also elevated in mice and pigs injected with NIL10. Mechanistically, NIL10 induced activation of the IL-10 receptor/STAT-3 signaling pathway, and STAT3-dependent inhibition of pro-inflammatory NF-kB transcription factor nuclear translocation, as evidenced by inhibition of nuclear p65 in macrophages stimulated with $500 \mathrm{mM}$ LPS in response to incubation with NIL10, and prevented by co-incubation with the STAT-3 pharmacological inhibitor STATTIC, as also evidenced by testing the NF-kB -dependent readout iNOS gene expression with same results.

Conclusions: Our findings propose NIL10 nanoparticles as a novel compound to improve cardiac function after myocardial infarction, to preventing cardiac necrosis by inducing polarization toward M2-resolving macrophages, through inhibition of STAT3-induced nuclear translocation of NF-kB.

\section{Introduction}

Cardiovascular diseases, including acute myocardial infarction (AMI), are the most frequent cause of death in the world [1]. AMI remains the commonest origin of heart failure (HF), which conditions longterm life quality and may end up to the death of the patient [2-4].

Following an ischemic event, the lack of oxygen and nutrients in the heart triggers an inflammatory response. Necrotic cardiomyocytes release signals that stimulate innate immune signaling, then cell mobilization to the damaged area begins, and finally, anti-inflammatory signaling leads to the replacement of necrotic myocardial tissue by the formation of a fibrotic scar [5]. Even though inflammation is a critical component of tissue healing, currently more contributions point towards a prolonged inflammatory response can compromise myocardial structure and function due to an adverse left ventricle remodelling, causing HF [6-8]. Therefore, targeting the inflammatory response may represent a promising strategy to prevent adverse outcomes after AMI [9]. 
Macrophages play homeostatic and immune control roles in myocardial remodeling throughout the process of inflammation [10] and polarize according to signals from their environment [11]. Altogether, in response to stimuli like bacterial lipopolysaccharide (LPS), interferon gamma (IFN- $\mathrm{y}$ ), or even hypoxic stimuli, M1 activated macrophages secrete pro-inflammatory cytokines, tumour necrosis factor (TNF-a), granulocyte colony-stimulating factor (G-CSF), granulocyte-macrophage colony-stimulating factor (GMCSF) motif chemokine ligand (CXCL) as CXCL10 and matrix metalloproteinases (MMPs) as MMP-2, MMP-9 and MMP-13. This population expand at the site of inflammation and change their phenotype serving as the first line of defence within hours-days. M2 resolving macrophages induced by antiinflammatory cytokines such as IL-4, IL-13, and IL-10, are capable of inducing fibroblast-mediated extracellular matrix production, cell proliferation and angiogenesis, leading to the repair of damaged tissues $[12,13]$. Mechanisms to promote polarization towards M2 state including backward prevention to M1 repolarization are essential to avoid adverse cardiac remodeling, in which the anti-inflammatory IL-10, is mainly involved in the process.

IL-10R is composed of two subunits, both of them have roles in signal transduction processes [14-16] which mainly induces anti-inflammatory responses, playing a key role in macrophage-mediated inflammation resolution. IL-10 binding to its receptor (IL10R) [17] results in activation of receptorassociated tyrosine kinases of Janus kinase family, JAK1 and TYK2, which in turn lead to phosphorylation of two tyrosine residues on IL-10R1. Phosphorylated IL-10R1 residues recruit STAT3 to the activated receptor, which causes the subsequent phosphorylation and activation of the latent transcription factor, phospholylated-STAT3 $[18,19]$.

The use of specific anti IL-10 antibodies is a successful approach to show the relevance of IL-10 during hindlimb reperfusion after femoral artery ligation [20], chronic inflammation [21], certain types of cancer $[22,23]$ and neuropatic pain [24]. Nevertheless, and to avoid possible antibody-induced immunogenicity responses, we designed lipid nanoparticles (NIL10) conjugated with IT9302 [25], a nanomeric peptide, homologous to the $\mathrm{C}$ terminus of IL-10, or a scramble peptide (NIL10SC) as a negative control, to evaluate its contribution to the resolution of inflammation following acute myocardial infarction in mice and pigs.

\section{Methods}

\section{Reagents and Equipment}

Hematoxylin-eosin (HE), Trichrome Masson staining reagents, Triphenyl tetrazolium chloride (TTC), Evans Blue and fetal bovine serum (FBS) were from Merck (St Louis, MO, USA). Horseradish peroxidase (HRP)conjugated anti-mouse secondary antibody and liquid 3,3'-diaminobenzidine (DAB) substrate were from Dako (Santa Clara, CA). Anti-IL10RA (ab225820) anti-MMP-9 (ab38898), anti-GAPDH (ab22555), anti- $\beta$ Tubulin (ab6046) antibodies were from Abcam (Cambridge, UK). Anti-NF-kB (sc-372) anti-NOS2 (sc-651), anti-IKB-a (sc-371), anti-EMMPRIN (sc-53064) antibodies were from Santa Cruz (Santa Cruz, CA). Goat anti-rabbit IgG Alexa Fluor 488 secondary antibody (A11008) and anti-P-IL10-RA (PA5-104991), 100- 
cell strainer (08-771-19), ACK Lysing Buffer (A1049201), anti-CD68 (11-0689-42) and anti-CD206 (122069-42) were from Thermo Fisher Scientific (Waltham, MA, US). Anti-STAT3 (79D7), anti-P-STAT3 (Y705, D3A7) and anti-P-IKB-a (S32, 14D4) antibodies were from Cell Signaling (Danvers, MA, US). Proteome Profiler Mouse Cytokine Array Kit (ARY006) and Proteome Profiler Human Cytokine Array Kit (ARY005B) were from R\&D Systems (Minneapolis, MN), ketamine was from Pfizer (New York, NY, US), isoflurane was from Abbvie (North Chicago, IL, US), propofol was from Fresenius (Bad Homburg, Germany), fentanyl was from Kern Pharma (Madrid, Spain), diazepam was from Roche (Basel, Switzerland), and the amiodarone was from Sanofi Aventis (Gentilly, France).

\section{Peptide and Nanoprobe composition}

Peptide composition of IT9302 and scrambled:

\section{IT9302: CAYMTMKIRN}

\section{IT9302-scrambled: CANYRMITKM}

The paramagnetic nanoparticles were prepared by the lipid film hydration method. In brief, a film was prepared by rotary evaporation of Gd-DTPA-bis (GdDTPA-BSA), 1,2-distearoyl-sn-glycero-3phosphoethanolamine-N-[ methoxy (polyethylene glycol)-2000] (DSPE-PEG2000), DSPE-PEG2000maleimide, and Rhodamine-PE, in a molar ratio 50:39:1:10, dissolved in a mixture of chloroform/methanol (4:1 v/v). The lipid film was hydrated with HEPES buffer, $\mathrm{pH} 6.7$, and $150 \mathrm{mM} \mathrm{NaCl}$, and the solution was rotated at $65^{\circ} \mathrm{C}$ for 1 hour. IT9302 and the corresponding scrambled peptides were modified by adding a terminal cysteine residue (Fig. 1) to bind to the maleimide moiety at a molar ratio 1:40 (micelle: peptide). Uncoupled peptides were separated with centrifuge concentrators of $100 \mathrm{kDa}$. The number of peptide molecules bound per micelle was calculated by HLPC, estimating $4 \pm 2$ and $5 \pm 1$ the number of peptides present in NIL10 and NIL10SC respectively. Physical and chemical properties, including Zeta-potential, nanoparticle size (calculated as hydrated diameter with dynamic laser light scattering (DSL, Malvern Zetasizer)), nanoparticle morphology (visualized with transmission microscopy (TEM)), longitudinal and transverse relaxivities, were calculated. Longitudinal relaxivities were evaluated by using inversion recovery sequences with 15 inversion times. Transverse relaxivities were calculated from spin-echo images and different echo times. In both cases, a series of images with different $\mathrm{T} 1$ and T2 weighting were generated. The $\mathrm{r} 1$ and $\mathrm{r} 2$ values were estimated from the slope of longitudinal and transverse relaxation rates vs Gd-DTPA or nanoparticle.

\section{Animal studies}

All the surgical procedures were performed in the Experimental Surgery Department of the Hospital Universitario La Paz in Madrid, in conforming to the Guide for the Care and Use of Laboratory Animals published by the US National Institutes of Health (NIH Publication No. 85-23, revised 1985), and the Animal Welfare Ethics Committee and complied with the EU Directive on experimental animals (63/2010 EU) and related Spanish legislation (RD 53/2013). PROEX 138/17.

\section{Porcine Model of Coronary Ischemia/Reperfusion}


All the surgical procedures were performed in the Experimental Surgery Department of the Hospital Universitario La Paz (Madrid, Spain) in conforming to the Guide for the Care and Use of Laboratory Animals published by the US National Institutes of Health (NIH Publication No. 85-23, revised 1985), and the Animal Welfare Ethics Committee and complied with the EU Directive on experimental animals (63/2010 EU) and related Spanish legislation (RD 53/2013).

20 male Yorkshire pigs ( $30 \pm 4 \mathrm{~kg}$ ) were housed 1 week preceding the surgery to avoid unease or stress associated with the new environment. Prior to the surgical intervention, animals were anesthetized with intramuscular ketamine $10 \mathrm{mg} / \mathrm{kg}$ and midazolam $0.5 \mathrm{mg} / \mathrm{kg}$. Anesthesia was induced by inhaled sevoflurane and maintained with continuous infusion of propofol $2 \mathrm{~mL} / \mathrm{kg} / \mathrm{h}$, fentanyl $50 \mu \mathrm{g} / \mathrm{kg} / \mathrm{h}$, and diazepam $10 \mu \mathrm{g} / \mathrm{kg} / \mathrm{h}$. After intubation and mechanical ventilation with $100 \%$ oxygen saturation, $5000 \mathrm{IU}$ of heparin and amiodarone $2 \mathrm{mg} / \mathrm{kg} / \mathrm{h}$ was administered to avoid blood clotting of catheters and malignant cardiac arrhythmias, respectively. Preceding the complete occlusion, hearts were submitted to ischemic preconditioning by blocking the LAD for short periods $(1,3$, and 5 minutes each).

Ischemia/reperfusion was produced by occluding LAD for 45 minutes using a JL3 6F catheter and an angioplasty balloon. The complete myocardial ischemia was confirmed by ST-segment elevation. Then, the balloon was removed to reopen the artery. Animals received NIL10 or NIL $10 S C(0.1 \mathrm{mg} / \mathrm{kg}) 24 \mathrm{~h}$ hours post-ischemia. Blood was extract before and after the procedure, 3 days post-ischemia and at final point. After 7 days of reperfusion, animals were sacrificed to extract the heart, spleen, kidney, lung, liver and pancreas. This samples were included in $4 \%$ formalin and other samples of the heart were immediately frozen at $-80^{\circ} \mathrm{C}$ to protein analyses.

\section{Murine Model of Coronary Ischemia/Reperfusion}

20 male twelve-week-old wild type, IL10 knock-out and IL10RA knock-out mice C57/BL6 were anesthetized by inhaled $3 \%$ sevoflurane and oxygen with a flow rate of $0.4 \mathrm{~L} / \mathrm{min}$ until loss of righting reflex. Then, endotracheal intubation was perform using an intubation cannula in order to carry out artificial ventilation (tidal volume: $260 \mu \mathrm{L} /$ stroke, ventilation rate: 130 strokes per minute). The fourth left intercostal space was opened and widened using chest mice retractors. Left ventricle was exposed and LAD was occluded for 30 min close by using a 6-0 silk suture and $1 \mathrm{~mm}$ tube. Reperfusion was performed by ligation release. After the procedure, the chest was closed, negative pressure restored, and the skin sutured. At the reperfusion time of 24 hours, NIL10 was venous-administrated. Animals were sacrificed at day 3,7 or 21 to extract the organs and process the samples. NIL10SC-treated animals were included in the assays as control, in which the same procedure was performed.

\section{Echocardiography}

Vivid Q ultrasound system from GE Healthcare (General Electric, Chicago, IL, USA), equipped with a 1.9-4 $\mathrm{MHz}$ scan head was used to determine LV function using a. Parasternal long and short-axis-view images of the heart were taken prior to the surgery, at the end of ischemia, and at the endpoint to determine LV function worsening and recovery. The parameters studied using the on-site software cardiac package were: systolic and diastolic interventricular septum thickness (IVS), systolic and diastolic left-ventricle 
internal diameter (LVID), systolic and diastolic left-ventricle posterior wall thickness (LVPW), left-ventricle ejection fraction (LVEF), left ventricle shortening fraction (LVFS), heart rate (HR), and cardiac output (CO). Data acquisition and analysis were performed by using the onsite cardiac software VividQ ultrasound equipment, and the same operator analyzed all samples to avoid the inter-observer biases.

\section{Histology}

Evans Blue/TTC staining in pigs: 7 days after surgery, the LAD was re-occluded through ballon inflation in the same location as day 0 , following the same method described before. Then, a $5 \mathrm{~F}$ fenestrated pigtail catheter was inserted through the second femoral artery and placed into the left ventricle to inject $200 \mathrm{~mL}$ of $5 \%$ Evans Blue solution to distribute the compound across the entire cardiovascular system, excepting the blood-deprived area of the heart. After 2 minutes, the animal was sacrificed, and the heart extracted and frozen at $-20^{\circ} \mathrm{C}$ for 24 hours. Then, hearts were cut into $0.8 \mathrm{~cm}$ slices and incubated in $1 \%$ TTC solution for 20 minutes at $37^{\circ} \mathrm{C}$ and next in $10 \%$ Paraformaldehyde solution. The necrotic area was calculated in reference to the area at risk to avoid inter-variability biasing.

Heart morphology was visualized by Hematoxylin/Eosin staining and collagen deposition was detected by Masson's trichrome staining both in mice and pigs.

\section{Confocal Microscopy}

Rhodamine containing NIL10 nanoparticles were visualized in 5- $\mu$ m heart sections mounted in PBS buffer, containing Hoechst for nuclei visualization by confocal microscopy.

RAW 264.7 cell monolayers were incubated with anti-NFkB primary antibody diluted 1:500 in PBS 1.5\% BSA for 1 hour, and then washed and incubated with the corresponding goat anti-rabbit IgG Alexa Fluor 488 secondary antibody diluted 1:1000 in PBS 1.5\% BSA for 1 hour. Cells were washed three times with PBS and mounted in PBS media, containing Hoechst for nuclei visualization. Images were taken using a Leica TCS SP5 confocal microscope. At least three different fields per condition were obtained.

\section{Immunoblotting}

Protein lysates from healthy and necrotic areas of mouse and pig hearts, were extracted with RIPA buffer to measure the expression of MMP-9, IL-10R, P-IL-10R, STAT3, and P-STAT3, while and P-IKB-a, and NOS2 were assayed in RAW247 cells. Proteins were separated by SDS-PAGE and transferred to PVDF membranes. Membranes were blocked with 3\% BSA in $25 \mathrm{mM}$ Tris, $150 \mathrm{mM} \mathrm{NaCl}, 0.05 \%$ Tween-20, pH 7.4 (T-TBS), washed and incubated with the corresponding primary antibodies diluted 1:1000 for one hour at room temperature. Membranes were subsequently washed 3 times with T-TBS and then incubated with horseradish peroxidase conjugated secondary antibodies 1:3000 for an hour for detection of proteins by chemiluminescence. The levels of GAPDH and $\beta$-Tubulin were used as loading controls.

\section{Blood collection and plasma isolation}

Animal blood samples were collected in buffer Sodium-Citrate tubes (363086) and EDTA tubes (367861) from a retro-orbital bleed in mice or from femoral venous in pigs respectively and were from BD 
Vacutainer (Franklin Lakes, NJ, US). Plasma was isolated from blood spun at 400g for 10 minutes. Plasma not used immediately was stored at $-20^{\circ} \mathrm{C}$.

\section{Cytokine and chemokine determinations}

We detected a total of 36 human cytokines, chemokines, and acute phase proteins simultaneously from the serum of pigs and mice at the times indicated, by using the Proteome Profiler Cytokine Array Kit (RD Systems, Minneapolis, MN) a membrane-based antibody array for the parallel determination of the relative levels of selected human cytokines and chemokines, as described [9].

\section{Single-cell suspension for flow cytometry}

Whole mouse hearts were mashed in complete DMEM (10\% FBS, penicillin/streptomycin) through a 100$\mu \mathrm{m}$ cell strainer. ACK Lysing Buffer was added to the single-cell suspension and spun down at $350 \mathrm{~g}$ for 5 minutes. The ACK was washed out with $50 \mathrm{~mL}$ of washing buffer (1\% FBS), and cells were incubated with 1:100 anti-EMMPRIN, anti-CD68 and anti-CD206 antibodies. All flow cytometry samples were assayed in a MACSQuant Analyzer Flow Cytometer and and analyzed with the Graphpad Prism software package.

\section{Statistical analysis}

All values were given as mean \pm S.D. Significance is reported at the $5 \%$ level. Whenever comparisons were made with a common control, significance of differences was tested by Dunnett's modification of the $t$ test.

\section{Results}

\section{NIL10 improves cardiac function in mice subjected to IR}

To study the specificity of nanoparticles, we first incubated NIL10 (Figure 1Af, B) in RAW 264.7 macrophages, detecting rhodamine-containing nanoparticles by confocal microscopy (Figure 1C). Colocalization with the IL-10R (as detected by immunohistofluorescence (FITC, green) was positive in RAW 264.7 cells incubated with NIL10 (rhodamine, red. Figure 1D), as detected by confocal microscopy (Merged panel, yellow and colocalization pixels panel), while no binding was found in cells incubated with NIL10SC (Figure 1E).

Kaplan Meier curves of healthy mice injected with 1, 10 and $100 \mathrm{mg} / \mathrm{kg}$ indicated no mortality linked to the lowest dose, whereas the dosages of 10 and $100 \mathrm{mg} / \mathrm{kg}$ were lethal for $20 \%$ and $25 \%$ of animals, respectively, after 30 days of testing (Figure 2A). To test for biodistribution, confocal microscopy visualization of NIL10 and NIL10SC in the heart, liver, kidney, pancreas, spleen and lungs of healthy animals revealed a faint accumulation of NIL10 in the heart, kidney and lungs (Figure 2B).

After selecting a dosage of $1 \mathrm{mg} / \mathrm{kg}$, we proceeded to inject $1 \mathrm{mg} / \mathrm{kg} \mathrm{NIL} 10$ or NIL10SC in mice 24 hours after IR (Figure 3A,B), resulting that NIL10 improved the LVEF by days 3 and 7 after IR $(41.5 \% \pm 4.33$ vs $68 \% \pm 7.02$ and $65.6 \% \pm 5.11$ respectively. Figure $3 \mathrm{C}$ ). Proof of the effect was obtained by performing the 
same assay in IL10 null mice, in which NIL10 also exhibited a significant degree of cardioprotection (Figure 3D). By contrast, in IL-10 receptor deficient mice subjected to IR, NIL10 had no effect (Figure 3E), suggesting that NIL10 induces cardiac protection at least, through activation of IL10 receptor signalling pathway.

\section{Administration of NIL10 reduces necrosis and fibrosis in mouse hearts subjected to IR}

To shed light on the underlying causes by which NIL10 induces cardioprotection, Hematoxilin/Eosin (H/E) staining of heart sections isolated by days 3 and 7 after IR, showed a significant reduction in the necrotic area in response to NIL10, when compared to the inflammatory foci and the extension of necrosis present in mice injected with NIL10SC (Figure 4). Likewise, the levels of the necrosis marker, matrix metalloproteinase 9 (MMP9), were markedly reduced in the necrotic areas of NIL10-injected mice (Figure $5 A$ ), including the extension of fibrosis, whereas in the NIL10SC group, fibrotic lesions were widespread in the hearts by day 7 after IR. (Figure 5B).

\section{NIL10 improves cardiac function in pigs subjected to IR}

To test whether NIL10 may also induce cardiac protection in large animals, we injected $1 \mathrm{mg} / \mathrm{kg} \mathrm{NIL10}$ or NIL10SC into pigs subjected to IR by angioplasty balloon inflation of the left anterior descending coronary artery (Figure 3A). As shown by Evans Blue/TTC staining of left ventricle sections (Figure 6A), after 7 days of IR we found a significant reduction of the necrotic area by $47 \%$ (NIL10SC $55 \pm 7.34$ vs NIL10 26.48 \pm 4.32 . Figure 6B), which contributes to explain the improvement in cardiac function as evidenced by the recovery of LVEF in animals injected with NIL10 (Figure 6C). As in mice, HE and Masson's trichrome staining of heart sections from pigs injected with NIL10, indicated a marked reduction of myocardial necrosis (Figure 6D) and fibrosis (Figure 6E), when compared with the NIL10SC group.

\section{Injection of NIL10 has an impact on macrophage polarization}

The observed differences in the number and severity of inflammatory foci led us to consider whether NIL10 might have an impact on macrophage polarization. Indeed, cell extracts obtained from the necrotic areas of mouse hearts indicated that after 3 days of IR, NIL10 promotes the presence of M2-resolving macrophages, while it takes 7 days to detect a similar effect when NIL10SC was injected (Figure 7A-B), which may help to explain the reduction of inflammatory foci in animals injected with NIL10.

We also assessed the level of 40 cytokines and chemokines in plasma collected from mice and pigs after 3 days of IR (Figure 8A). We identified differences in the expression of a significant number of cytokines, highlighting the presence of 4 mouse (5 pig) expression clusters [26] depending on whether the animals have been injected with NIL10 or NIL10SC, highlighting that in both species, we identified a marked 
increase in the expression of anti-inflammatory cytokines IL-4, $-10,-13,-16$, and -27 , together with IL-5, and IL-7 in pigs, in response to NIL10 administration (Figure 8BC). Taken together, these results suggest that NIL10 may induce cardiac protection by at least, macrophage polarization towards inflammation resolution.

\section{NIL10 induces phosphorylation of STAT3 in pigs subjected to IR.}

Cardiac exposure to ischemia triggers activation of specific pro-inflammatory transcription factors, of which NF-KB plays a major role [27]. Polarization of immune cells through an inflammatory resolving state implies the activation of specific signaling cascades, highlighting the contribution of IL-10-induced JNK signaling pathway that activates STAT3 transcriptional regulation of anti-inflammatory cascades, including prevention of NF-KB nuclear translocation [28].

NIL10 led to accumulation of phosphorylated IL-10 receptor subunit IL-10RA and phospho-STAT3 in mice (Figure 9) and in pigs subjected to IR (Figure 10), indicative that NIL10 induces receptor-agonist complex activation through the IL10-JNK-STAT-3 signalling pathway.

The anti-inflammatory effect NIL10 was further investigated in RAW 264.7 macrophages stimulated with $500 \mu \mathrm{M}$ LPS, in which nuclear translocation of pro-inflammatory NF- $\mathrm{KB}$ transcription factor was prevented by NIL10 (Figure 10A), at least by suppressing phosphorylation of the NF- KB cytoplasmic sequestering IKB-a (Figure 10B), required for IKB-a proteasome-mediated proteolytical degradation.

To further validate the anti-inflammatory effect of NIL10, we stimulated NF- KB nuclear translocation with $500 \mu \mathrm{M}$ LPS in RAW 264.7 cells co-inubated with $5 \mu \mathrm{M}$ of STAT3-specific pharmacological inhibitor STATTIC, which prevented NIL10-induced cytoplasmic localization of NF-KB in LPS-treated cells, indicating that NIL10 inhibits NF- KB nuclear translocation at least, by STAT-3 activation (Figure 10C). The expression of the readout inducible nitric oxide synthase (iNOS) by pro-inflammatory signals was also assayed. As shown, iNOS was expressed by stimulating RAW 264.7 macrophages with $500 \mu \mathrm{M}$ LPS, and significantly inhibited by co-incubation with NIL10, while the effect was reversed in the presence of STATTIC (Figure 11 lower panels). Taken together, NIL10 may act as anti-inflammatory effector through IL-10/STAT3 signaling pathway in myocardial ischemia/reperfusion.

\section{Discussion}

In the current work we show that NIL10, a novel anti-inflammatory nanoparticle, induces cardiac protection after acute myocardial infarction following cardiac ischemia/reperfusion. Administration of NIL10 24 hours after IR, attenuated interstitial fibrosis and improved LVEF in mice and pigs by days 3 and 7 after IR. The same pattern applied to IL10 knockout mice, while in IL-10 receptor deficient mice, NIL10 did not have an effect on cardiac recovery. As shown by confocal microscopy, NIL10 binds to IL10R, inducing accumulation of $\mathrm{M} 2$ macrophage populations after 3 days of IR, in which anti-inflammatory cytokines IL-4, IL-7, IL-10, IL-13, IL-16 and IL-27 were increased, at least through STAT-3 dependent inhibition of NF-KB nuclear translocation. 
IL-10 is an anti-inflammatory cytokine that leads to cardiovascular protection in human atherosclerosis [29], acute coronary syndrome [30], unstable angina [31], and heart failure [32]. IL-10 plays an important role in the final outcome of myocardial infarction. A positive correlation between serum IL-10 levels with LVEF in patients after myocardial infarction, suggest the implication of IL-10 in predicting acute and chronic heart failure [33], as shown in patients with metabolic syndrome, in which higher IL-10 serum levels are associated with lower incidence of severe coronary artery disease [34[30]]. Here we provide with a novel compound based on the anti-inflammatory properties of IL-10, in which both in mice and pigs, it has been shown to prevent cardiac necrosis after myocardial ischemia/reperfusion.

It has been described the benefits of using small molecules, including oligopeptides, acting as agonist of several proteins. We previously described the cardioprotective effect of intravenous administration of NAP9, nanoparticles carrying AP9, a small peptide which specifically binds to EMMPRIN, a protein involved in the extracellular matrix degradation during myocardial infarction in mice and pigs subjected to myocardial IR [35]. Others have found that synthetic IL-10 analogue peptide IT9302 can interact to IL10 receptor and mimic its anti-inflammatory effect both in vitro in human melanoma cells [36], and in monocyte differentiation to TGF- $\beta$ tolerogenic dendritic cells [25] and in rabbits subjected with and acute pancreatitis [37] and acute lung injury [38]. However, the benefits of IT9302 in other inflammatory pathologies were so far unknown.

The use of several types of peptides in rodent models of myocardial infarction has been widely described. Examples include the use of Tat-DAXXp, a fusion peptide with Tat cell penetrating peptide with the death associated protein peptide [39], a synthetic apolipoprotein A-I mimetic peptide 4F [40], the Edomain region of the human MGF protein [41], or UM206, a peptide derived from regions of high homology between Wnt proteins [42]. Each example is a promising strategy for preventing progression of disease in rodents, although no studies have yet been performed in large animal models, such as pigs, to validate the strategy in patients suffering myocardial infarction. In the current work we provided the efficacy of using a new IT9302 conjugated nanoparticle, both in mice and in pigs with similar results.

Currently, the efficacy of specific treatments after myocardial infarction are limited for reasons that include lack of specific biodistribution, reduced half-life, or even toxicity issues. Given their small size, many therapeutic strategies have used nanoparticles conjugated with the therapeutic principle, which enables to freely travel throughout vasculature reaching the target faster, and thanks to their lipid composition makes them biocompatible with very little chance of rejection [43]. Furthermore, nanoparticles are extremely versatile, as additional components can be incorporated into their composition to enable the acquisition of new functionalities. Such is the case of NIL10 and NAP9 [35] as mentioned above, both nanoparticles based on lipid micelles, incorporating a fluorochrome in their composition to gain visualization by fluorescence. In addition, we have incorporated gadolinium to its formulation, which has enabled us to make an advance towards in vivo non-invasive visualization by nuclear magnetic resonance [35]. 
IL-10 triggers a downstream signalling pathway through binding to IL-10 receptors which phosphorylates JAK1, and further phosphorylates the intracellular domain of IL-10R-a, needed for the recruitment and phosphorylation of STAT-3, which at the end it regulates gene expression of anti-inflammatory genes [44]. Interestingly, others found that IL-10 functional domain homolog IT9302 induced monocyte differentiation to TGF- $\beta$ tolerogenic dendritic cells by an independent JNK/STAT-3 signalling pathway [36]. However, our results point to STAT-3 activation as a mechanism induced by NIL10 in cardiac protection against myocardial infarction. This apparent contradiction may lie in the mechanism of IT9302 administration, which in the case of NIL10 it specifically binds to IL-10 receptor, as shown by confocal microscopy, when compared to its negative control NIL10SC (Figure 1). Indeed, in IL-10 receptor null mice subjected to myocardial IR, administration of NIL10 had no effect (Figure 3).

The anti-inflammatory effect of IL-10 is in addition driven by STAT-3-mediated induction of several mediators including certain transcription factors such as ETV3, and the Strawberry notch homologue-2, that inhibit NF- KB mediated transcription in LPS stimulated macrophages [28]. Here, we also found that NIL10 induced STAT-3-NF-KB inhibition in LPS-stimulated RAW 264.7 macrophages, as confirmed by the reversion of NIL10-mediated inhibition of iNOS expression in LPS-stimulated cells by pharmacological inhibition of STAT-3 in this context.

\section{Conclusions}

In conclusion, we provide a feasible delivery of a novel compound to target the inflammatory response after acute myocardial infarction and reperfusion, representing a reliable strategy to preserve heart contractility, as a mechanism to prevent progression of myocardial necrosis. In addition to NIL10, our approach supports the use of additional peptides against specific targets susceptible to inhibition in the acute phase of heart failure post myocardial infarction. Further studies to test the long-term effects of NIL10 administration in pigs will be a key step to initiate validation in the clinical setting.

\section{Declarations}

\section{Ethics approval and consent to participate}

Not applicable

\section{Consent for publication}

Not applicable

\section{Availability of data and materials}

Most of the datasets supporting the conclusions of this work are included in the current article. The datasets are available on reasonable request.

\section{Competing interest}


The authors declare that they have no competing interests

\section{Funding}

This work was supported by the following grants: MINECO/AEI/FEDER/EU “Investigación orientada a los retos de la sociedad", co-founded by European Regional Development Fund/European Social Fund. A way to achieve Europe (SAF2017-87342-R, CZ). MICINN/AEI/FEDER/EU “I+D+i ORIENTADA A LOS RETOS DE LA SOCIEDAD, Co-funded by European Regional Development Fund/European Social Fund; "A way to make Europe" (PID2020-118371RB-I00, CZ). MICINN AEI/FEDER/EU “Prueba de Concepto” Co-funded by European Regional Development Fund/European Social Fund; "A way to make Europe" (PDC2021121817-I00, CZ).

\section{Author's contributions}

C.Z. designed the study, analyzed, interpreted data, and prepared the manuscript; L.T. and L.B. performed experiments and interpreted data; I.H., R.R-C. B.J-G., N.A. and J.D-M. performed experiments. K.O-P. and M.F., synthesized nanoparticles. M.S. and JL. Z. provided manuscript editing and interpreted the findings.

\section{Acknowledgments}

We want to acknowledge Dr. Carlota Largo Aramburu for her outstanding technical support.

\section{References}

1. Virani SS, Alonso A, Benjamin EJ, Bittencourt MS, Callaway CW, Carson AP, et al. Heart disease and stroke statistics-2020 update: A report from the American Heart Association. Circulation. 2020;141:e139-596.

2. Velagaleti RS, Pencina MJ, Murabito JM, Wang TJ, Parikh NI, et al. Long-term trends in the incidence of heart failure after myocardial infarction. Circulation. 2008;118:2057-62.

3. Chen J, Hsieh AFC, Dharmarajan K, Masoudi FA, Krumholz H. M. National trends in heart failure hospitalization after acute myocardial infarction for medicare beneficiaries 1998-2010. Circulation. 2013;128:2577-84.

4. Ezekowitz JA, Kaul P, Bakal JA, Armstrong PW, Welsh RC, McAlister FA. Declining In-Hospital Mortality and Increasing Heart Failure Incidence in Elderly Patients With First Myocardial Infarction. $J$ Am Coll Cardiol. 2009;53:13-20.

5. Prabhu SD, Frangogiannis NG. The biological basis for cardiac repair after myocardial infarction. Circ Res. 2016;119:91-112.

6. Frangogiannis N. Targeting the Inflammatory Response in Healing Myocardial Infarcts. Curr Med Chem. 2006;13:1877-93.

7. Westman PC, Lipinski MJ, Luger D, Waksman R, Bonow RO, Wu E, et al. Inflammation as a Driver of Adverse Left Ventricular Remodeling after Acute Myocardial Infarction. J Am Coll Cardiol. 
2016;67:2050-60.

8. Peet C, Ivetic A, Bromage DI, Shah AM. Cardiac monocytes and macrophages after myocardial infarction. Cardiovasc Res. 2020;116:1101-12.

9. Ramirez-Carracedo R, Tesoro L, Hernandez I, Diez-Mata J, Piñeiro D, Hernandez-Jimenez M, et al. TargetingTLR4 with aptoll improves heart function in response to coronary ischemia reperfusion in pigs undergoing acute myocardial infarction. Biomolecules. 2020;10:1167.

10. Fujiu K, Wang J, Nagai R. Cardioprotective function of cardiac macrophages. Cardiovasc Res. 2014;102:232-9.

11. Martinez FO, Gordon S. The M1 and M2 paradigm of macrophage activation: Time for reassessment. F1000Prime Rep. 2014. 6:13.

12. Sica A, Mantovani A. Macrophage plasticity and polarization: In vivo veritas. J Clin Invest. 2012;122:787-95.

13. Weber JS, Gibney G, Sullivan RJ, Sosman JA, Slingluff CL Jr, Lawrence DP, et al. Macrophage Activation and Polarization: Nomenclature and Experimental Guidelines. Immunity. 2014;41:14-20.

14. Tedgui A, Mallat Z. Anti-inflammatory mechanisms in the vascular wall. Circ Res. 2001;88:877-87.

15. Van Der Meeren A, Squiban C, Gourmelon P, Lafont H, Gaugler MH. Differential regulation by IL-4 and IL-10 of radiation-induced IL- 6 and IL-8 production and ICAM-1 expression by human endothelial cells. Cytokine. 1999;11:831-8.

16. Selzman CH, Mclntyre RC Jr, Shames BD, Whitehill TA, Banerjee A, Harken AH. Interleukin-10 inhibits human vascular smooth muscle proliferation. J Mol Cell Cardiol. 1998;30:889-96.

17. Josephson K, Logsdon NJ, Walter MR. Crystal structure of the IL-10/IL-10R1 complex reveals a shared receptor binding site. Immunity. 2001;15:35-46.

18. Weber-Nordt RM, Riley JK, Greenlund AC, Moore KW, Darnell JE, Schreiber RD. Stat3 recruitment by two distinct ligand-induced, tyrosine- phosphorylated docking sites in the interleukin-10 receptor intracellular domain. J Biol Chem. 1996;271:27954-61.

19. Riley JK, Takeda K, Akira S, Schreiber RD. Interleukin-10 Receptor Signaling through the JAK-STAT Pathway. J Biol Chem. 1999;274:16513-21.

20. Götze AM, Schubert C, Jung G, Dörr O, Liebetrau C, Hamm CW, et al. IL10 Alters Peri-Collateral Macrophage Polarization and Hind-Limb Reperfusion in Mice after Femoral Artery Ligation. Int J Mol Sci. 2020;21:2821.

21. Zaringhalam J, Akhtari Z, Eidi A, Ruhani AH, Tekieh E. Relationship between serum IL10 level and p38MAPK enzyme activity on behavioral and cellular aspects of variation of hyperalgesia during different stages of arthritis in rats. Inflammopharmacology. 2014;22:37-44.

22. Qian Q, Wu C, Chen J, Wang W. Relationship between IL10 and PD-L1 in Liver Hepatocellular Carcinoma Tissue and Cell Lines. Biomed Res Int. 2020;16:8910183.

23. Hsu TI, Wang YC, Hung CY, Yu CH, Su WC. Chang WCet al. Positive feedback regulation between IL10 and EGFR promotes lung cancer formation. Oncotarget. 2016;7:20840-54. 
24. McKelvey R, Berta T, Old E, Ji RR, Fitzgerald M. Neuropathic pain is constitutively suppressed in early life by anti-inflammatory neuroimmune regulation. J Neurosci. 2015;35:457-66.

25. López MN, Pesce B, Kurte M, Pérez C, Segal G, Roa J, et al. A synthetic peptide homologous to IL-10 functional domain induces monocyte differentiation to TGF- $\beta$ + tolerogenic dendritic cells. Immunobiology. 2011;216(10):1117-26.

26. Babicki S, Arndt D, Marcu A, Liang Y, Grant JR, Maciejewski A, et al. Heatmapper: web-enabled heat mapping for all. Nucleic Acids Res. 2016;44:W147-53.

27. Shimamoto A, Chong AJ, Yada M, Shomura S, Takayama H, Fleisig AJ, et al. Inhibition of Toll-like receptor 4 with eritoran attenuates myocardial ischemia-reperfusion injury. Circulation. 2006;114(1 Suppl):I270-4.

28. Saraiva M, Vieira P, O'Garra A. Biology and therapeutic potential of interleukin-10. J Exp Med. 2020;217(1):e20190418.

29. Ambrosius W, Kazmierski R, Michalak S, Kozubski W. Anti-inflammatory cytokines in subclinical carotid atherosclerosis. Neurology. 2006;66:1946-8.

30. Heeschen C, Dimmeler S, Hamm CW, Fichtlscherer S, Boersma E, Simoons ML, et al. CAPTURE Study Investigators. Serum level of the antiinflammatory cytokine interleukin-10 is an important prognostic determinant in patients with acute coronary syndromes. Circulation. 2003;107:2109-14.

31. Smith DA, Irving SD, Sheldon J, Cole D, Kaski JC. Serum levels of the antiinflammatory cytokine interleukin-10 are decreased in patients with unstable angina. Circulation. 2001;104:746-9.

32. Miettinen KH, Lassus J, Harjola VP, Siirilä-Waris K, Melin J, Punnonen KR, et al. Prognostic role of proand anti-inflammatory cytokines and their polymorphisms in acute decompensated heart failure. Eur J Heart Fail. 2008;10:396-403.

33. Lakhani HV, Khanal T, Gabi A, Yousef G, Alam MB, Sharma D, et al. Developing a panel of biomarkers and miRNA in patients with myocardial infarction for early intervention strategies of heart failure in West Virginian population. PLoS One. 2018;13:e0205329.

34. Barcelos ALV, de Oliveira EA, Haute GV, Costa BP, Pedrazza L, Donadio MVF, et al. Association of IL-10 to coronary disease severity in patients with metabolic syndrome. Clin Chim Acta. 2019;495:394-8.

35. Cuadrado I, Piedras MJ, Herruzo I, Turpin Mdel C, Castejón B, Reventun P, et al. EMMPRIN-Targeted magnetic nanoparticles for in vivo visualization and regression of acute myocardial infarction. Theranostics. 2016;6:545-57.

36. Kurte M, López M, Aguirre A, Escobar A, Aguillón JC, et al. A synthetic peptide homologous to functional domain of human IL-10 down-regulates expression of MHC class I and Transporter associated with Antigen Processing 1/2 in human melanoma cells. J Immunol. 2004;173:1731-7.

37. Osman MO, Gesser B, Mortensen JT, Matsushima K, Jensen SL, et al. Profiles of pro-inflammatory cytokines in the serum of rabbits after experimentally induced acute pancreatitis. Cytokine. 2002;17:53-9.

38. Osman MO, Jacobsen NO, Kristensen JU, Deleuran B, Gesser B,et al. IT 9302, a synthetic interleukin10 agonist, diminishes acute lung injury in rabbits with acute necrotizing pancreatitis. Surgery. 
1998;124:584-92.

39. Boisguérin P, Covinhes A, Gallot L, Barrère $C$, Vincent A, et al. A novel therapeutic peptide targeting myocardial reperfusion injury. Cardiovasc Res. 2020;116:633-44.

40. Moreira RS, Irigoyen MC, Capcha JMC, Sanches TR, Gutierrez PS, et al. Synthetic apolipoprotein A-I mimetic peptide $4 \mathrm{~F}$ protects hearts and kidneys after myocardial infarction. Am J Physiol Regul Integr Comp Physiol. 2020;318:R529-44.

41. Peña JR, Pinney JR, Ayala P, Desai TA, Goldspink PH. Localized delivery of mechano-growth factor Edomain peptide via polymeric microstructures improves cardiac function following myocardial infarction. Biomaterials. 2015;46:26-34.

42. Laeremans $H$, Hackeng TM, van Zandvoort MA, Thijssen VL, Janssen BJ, et al. Blocking of frizzled signaling with a homologous peptide fragment of wnt3a/wnt5a reduces infarct expansion and prevents the development of heart failure after myocardial infarction. Circulation. 2011;124:162635.

43. MacRitchie N, Di Francesco V, Ferreira MFMM, Guzik TJ, Decuzzi P,et al. Nanoparticle theranostics in cardiovascular inflammation. Semin Immunol. 2021;30:101536.

44. Riley JK, Takeda K, Akira S, Schreiber RD. Interleukin-10 receptor signaling through the JAK-STAT pathway. Requirement for two distinct receptor-derived signals for anti-inflammatory action. J Biol Chem. 1999;274:16513-21.

\section{Figures}




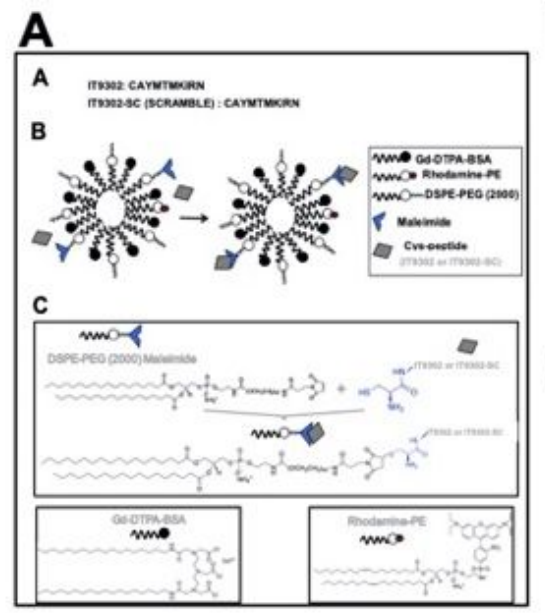

B

Figure 1

\begin{tabular}{|c|c|c|c|c|c|}
\hline SAMPLE & $\begin{array}{c}\text { Hydrodynamic } \\
\text { Size }\end{array}$ & z-potential & $\begin{array}{c}\mathbf{r}_{1} \\
\left(\mathbf{s}^{-1} \mathbf{m M}^{-1}\right)\end{array}$ & $\begin{array}{c}\mathbf{r}_{2} \\
\left(\mathbf{s}^{-1} \mathbf{m M}^{-1}\right)\end{array}$ & $\mathbf{r}_{2} / \mathbf{r}_{1}$ \\
\hline Micelle & $\begin{array}{c}111,5 \mathrm{~nm} \text { (PDI: } \\
0.253)\end{array}$ & $-28.9 \mathrm{mV}$ & 28.93 & 55.21 & 1.91 \\
\hline NIL-10 & $\begin{array}{c}135 \mathrm{~nm}(\text { PDI: } \\
0.280)\end{array}$ & $-22,7 \mathrm{mV}$ & 18 & 39.07 & 2.17 \\
\hline NIL-10-SC & $\begin{array}{c}132.6 \mathrm{~nm}(\text { PDI: } \\
0.263)\end{array}$ & $-26.7 \mathrm{mV}$ & 19.53 & 46.77 & 2.39 \\
\hline
\end{tabular}

C

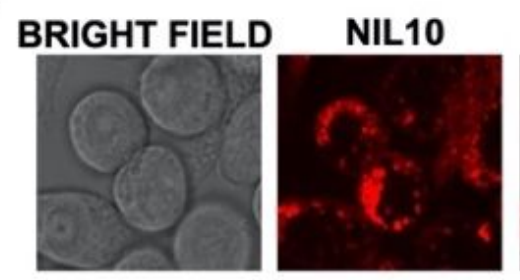

MERGED

D
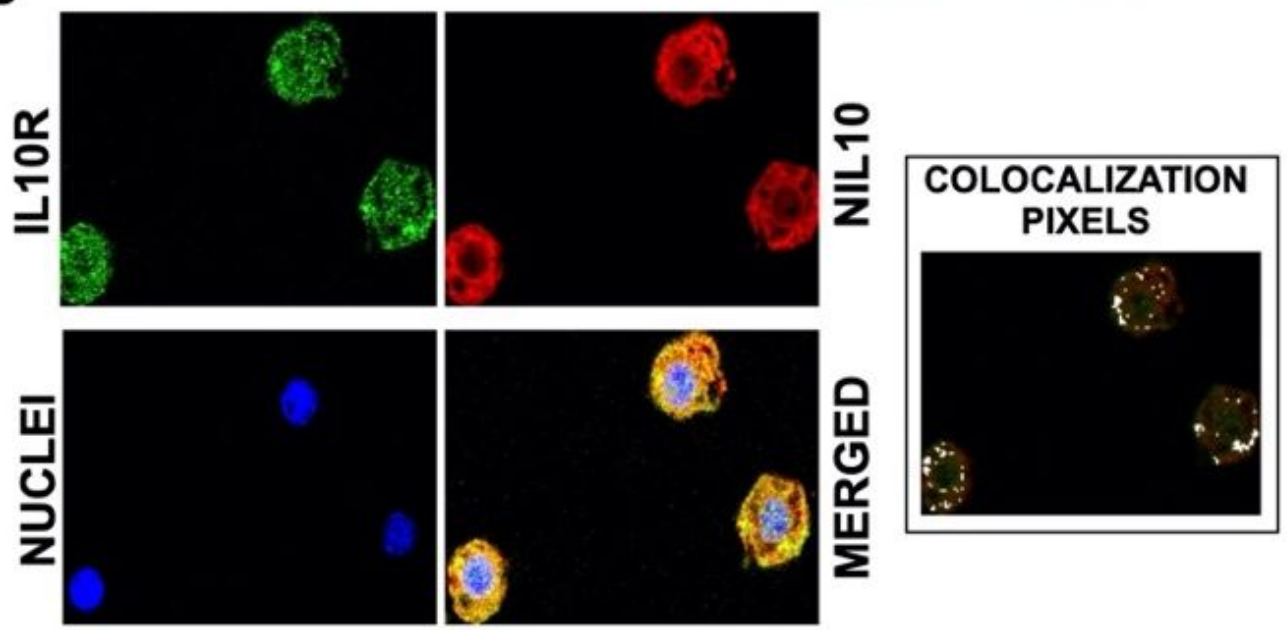

$\Sigma$

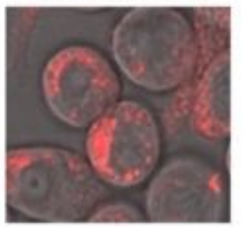

E
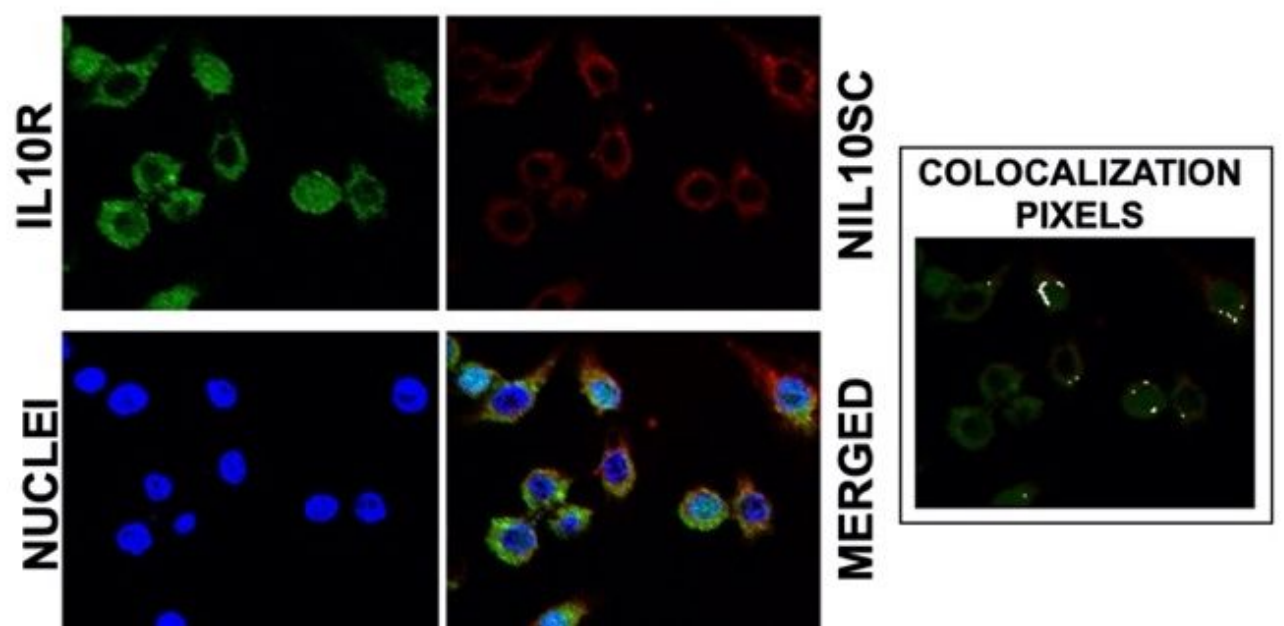

Figure 1

NIL10 binds to IL-10R in RAW 264.7 cells. A. Nanoparticle composition of NIL10 and NIL10SC. B. Zpotential, longitudinal and transversal relaxivities of NIL10 and NIL10SC nanoparticles. C. Bright field and confocal microscopy sections of RAW 264.7 macrophages, detecting rhodamine (red) containing NIL10 nanoparticles. D. Colocalization of NIL10 (rhodamine, red) with IL-10R (FICT, green) in confocal microscopy sections of RAW 264.7 cells incubated with NIL10 and anti-IL-10R specific antibody. Merged 
panel show co-localization of both signals. Nuclei were stained with Hoecht. Right panel: colocalization analysis with Image $\mathrm{J}$ co-localization plugin software, in which white dots correspond to co-localization $(\mathrm{N}=3)$ E. Colocalization of NIL10SC (rhodamine, red) with IL10R (FICT, green) in confocal microscopy sections of RAW 264.7 cells incubated with NIL10SC and anti-IL-10R specific antibody. Merged panel show co-localization of both signals. Nuclei were stained with Hoecht. Right panel colocalization analysis with Image $\mathrm{J}$ co-localization plugin software, in which white dots correspond to co-localization $(\mathrm{N}=3)$. 
Figure 2

A

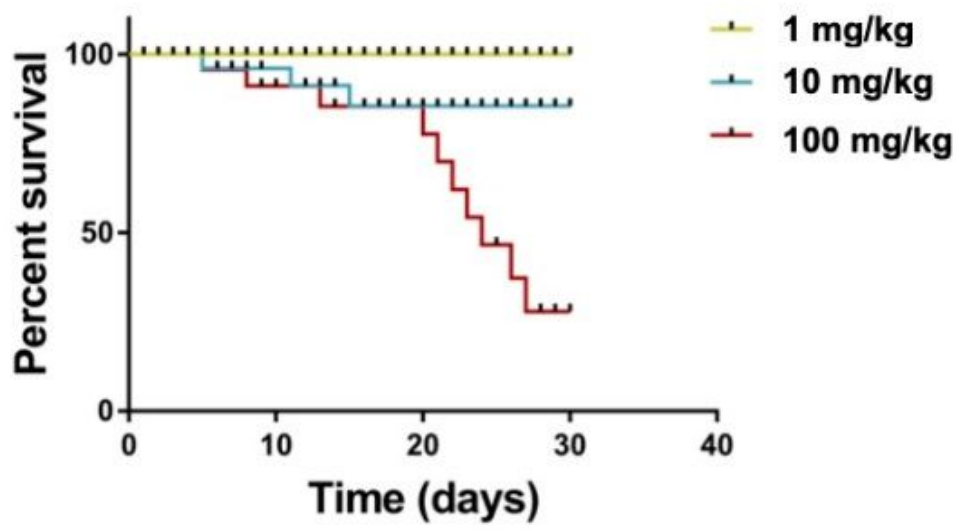

B

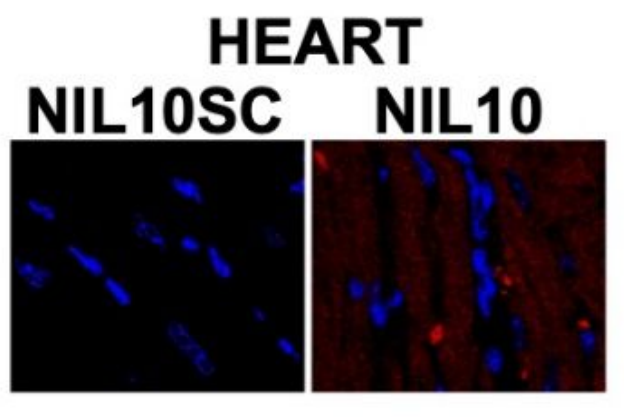

KINDEY

NIL10SC NIL10
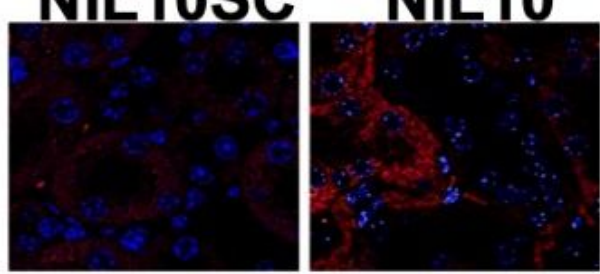

SPLEEN

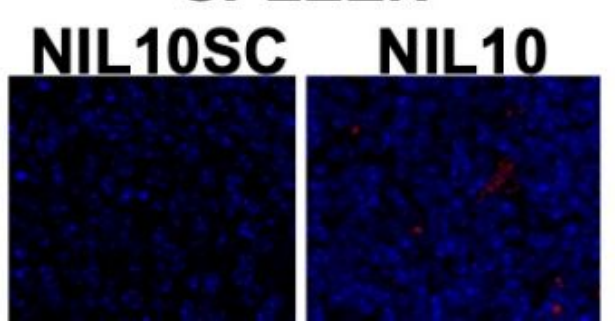

LIVER

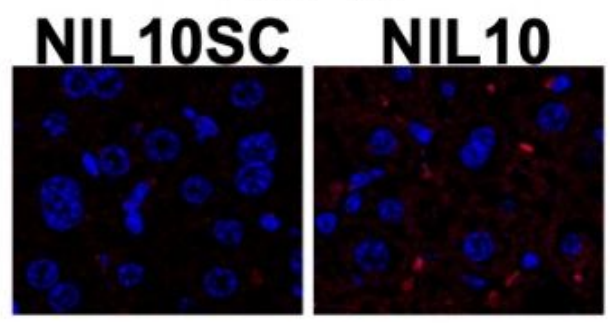

PANCREAS

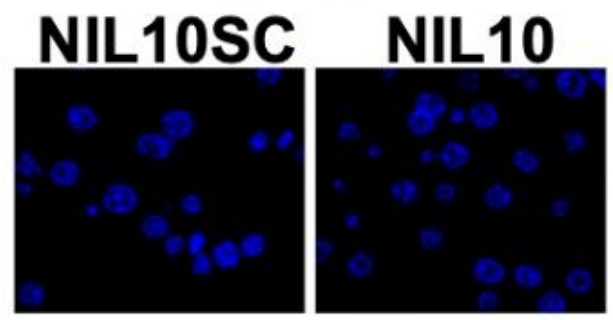

LUNG

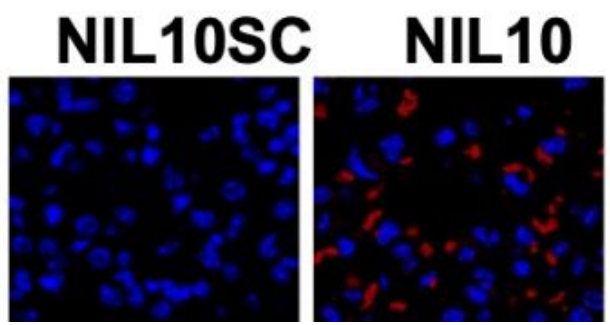

Figure 2

Dose effect of NIL10 on animal survival and organ biodistribution. A. Kaplan Meier curve showing the percentage of survival of healthy pigs injected with the doses indicated of NIL10 ( $\mathrm{N}=10$ mice/condition). B. Confocal microscopy detection of NIL10 and NIL10SC in heart, liver, kidney pancreas, spleen and lung sections of healthy animals ( $\mathrm{N}=10$ mice/group). 


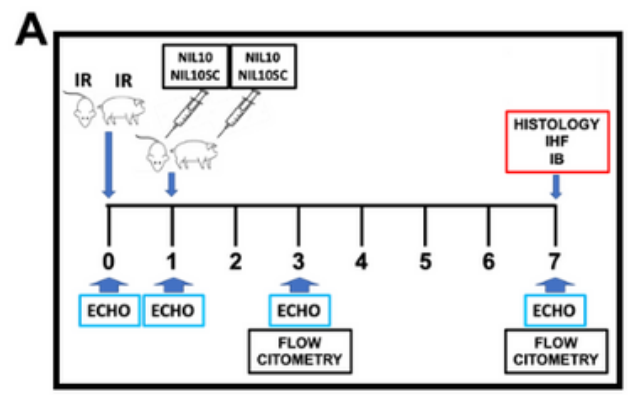

B

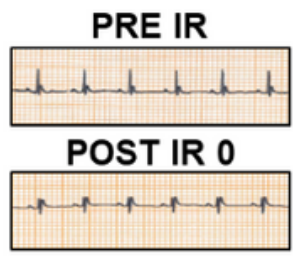

IL10 NULL MICE

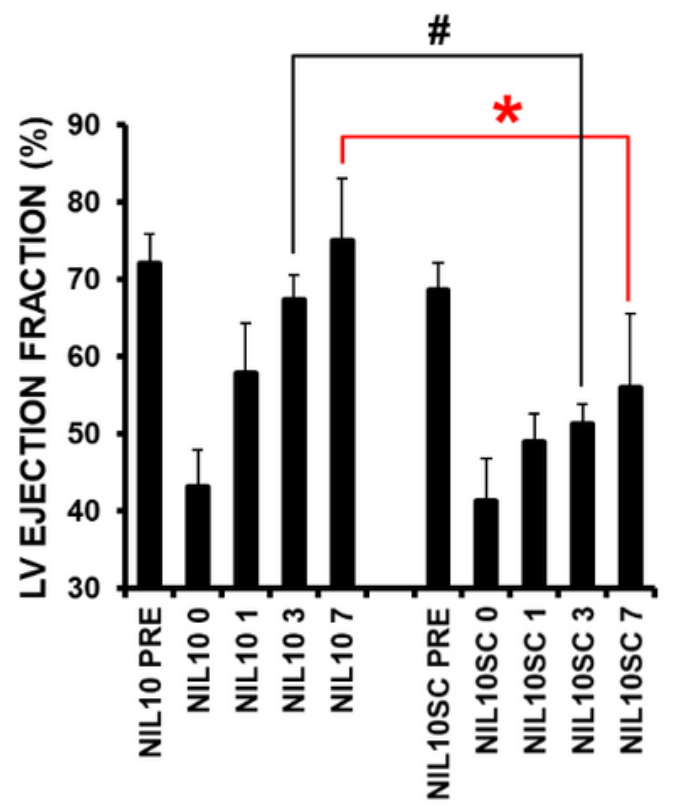

Figure 3

C

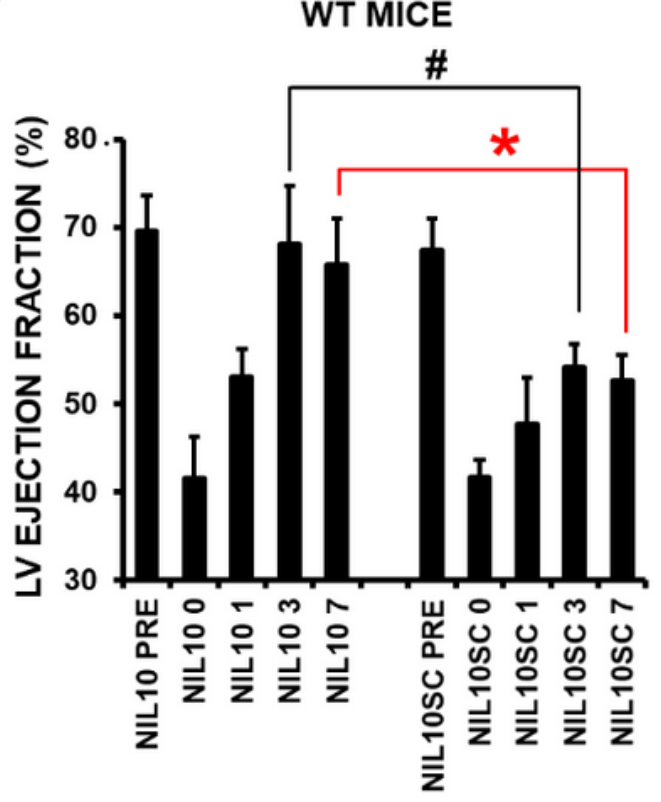

E

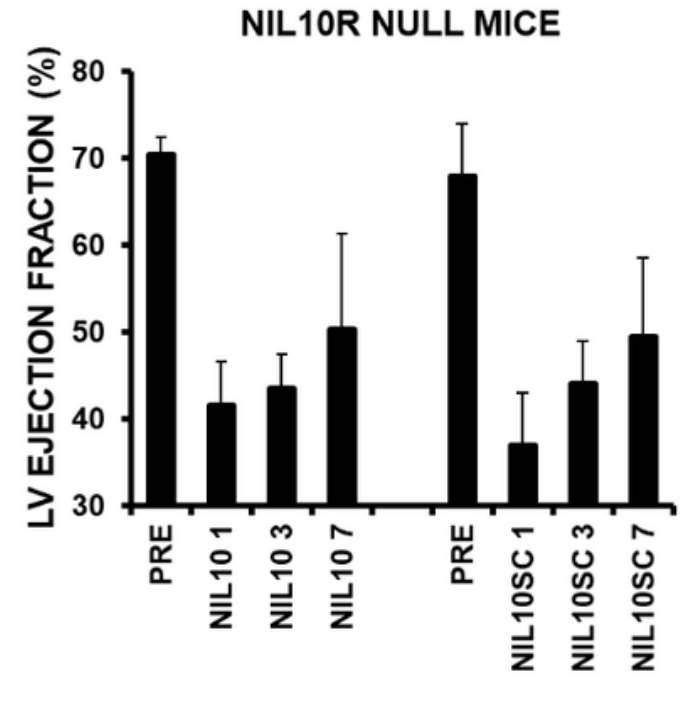

Figure 3

NIL10 induces cardiac protection in mice subjected to IR. A. Outline of the proceedings performed. B. Representative electrocardiogram, showing ST-elevation after LAD occlusion. C. Left ventricle ejection fraction (LVEF) of wild type mouse hearts at the times indicated after IR and injected with NIL10 or NIL10SC as in A. N=10 mice/group. Results expressed as mean \pm SD. ${ }^{*} \mathrm{P}<0.03$ NIL10 vs NIL10SC day 7. \#P<0.05 NIL10 vs NIL10SC day 3. D. LVEF in IL-10 knockout mice. N=10 mice/group. Results expressed 
as mean \pm SD. ${ }^{*} P<0.01$ NIL10 vs NIL10SC day 7. \#P<0.05 NIL10 vs NIL10SC day 3. E. LVEF in IL-10 receptor knockout mice. $\mathrm{N}=10$ mice/group. Results expressed as mean $\pm \mathrm{SD}$.

Figure 4

A

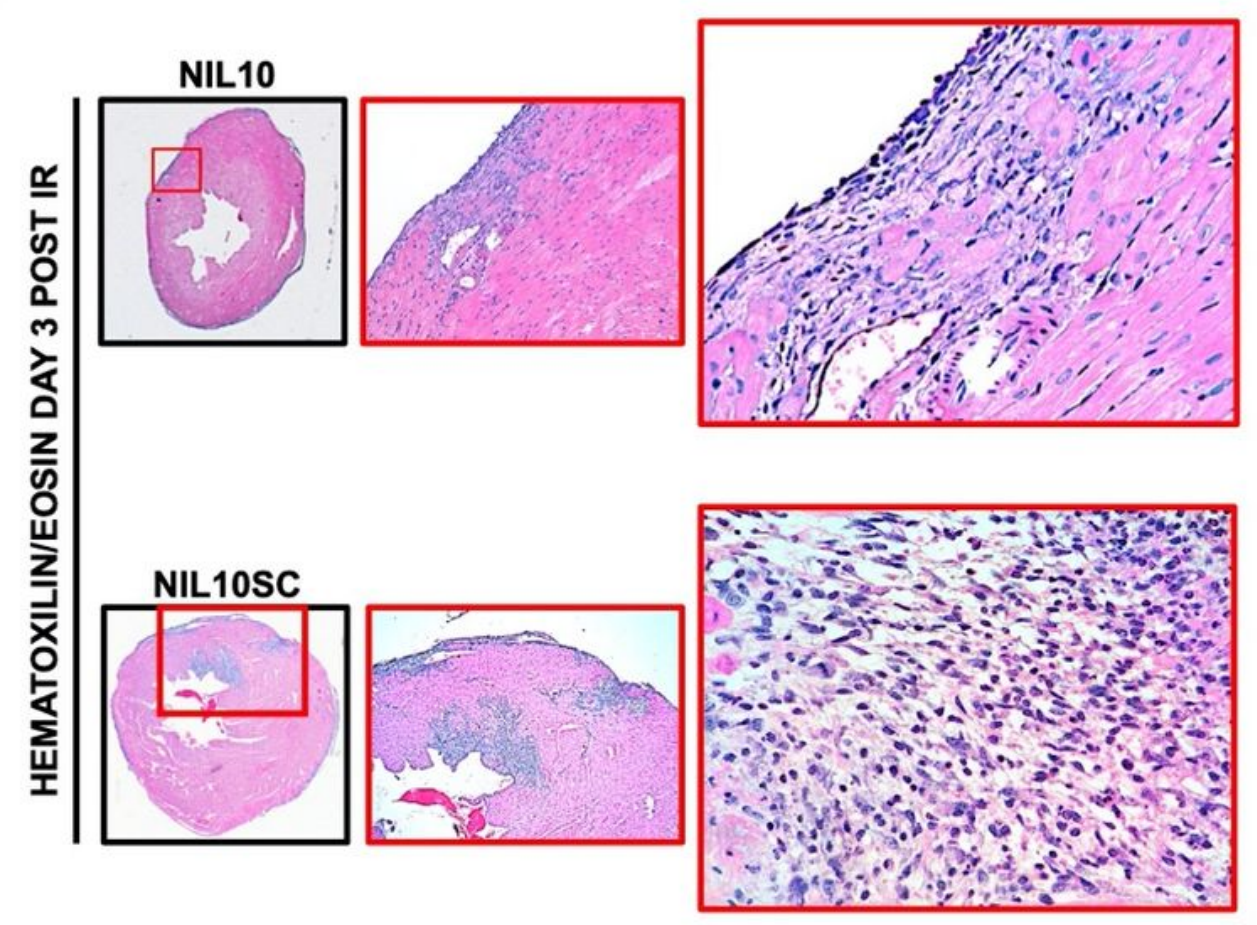

B
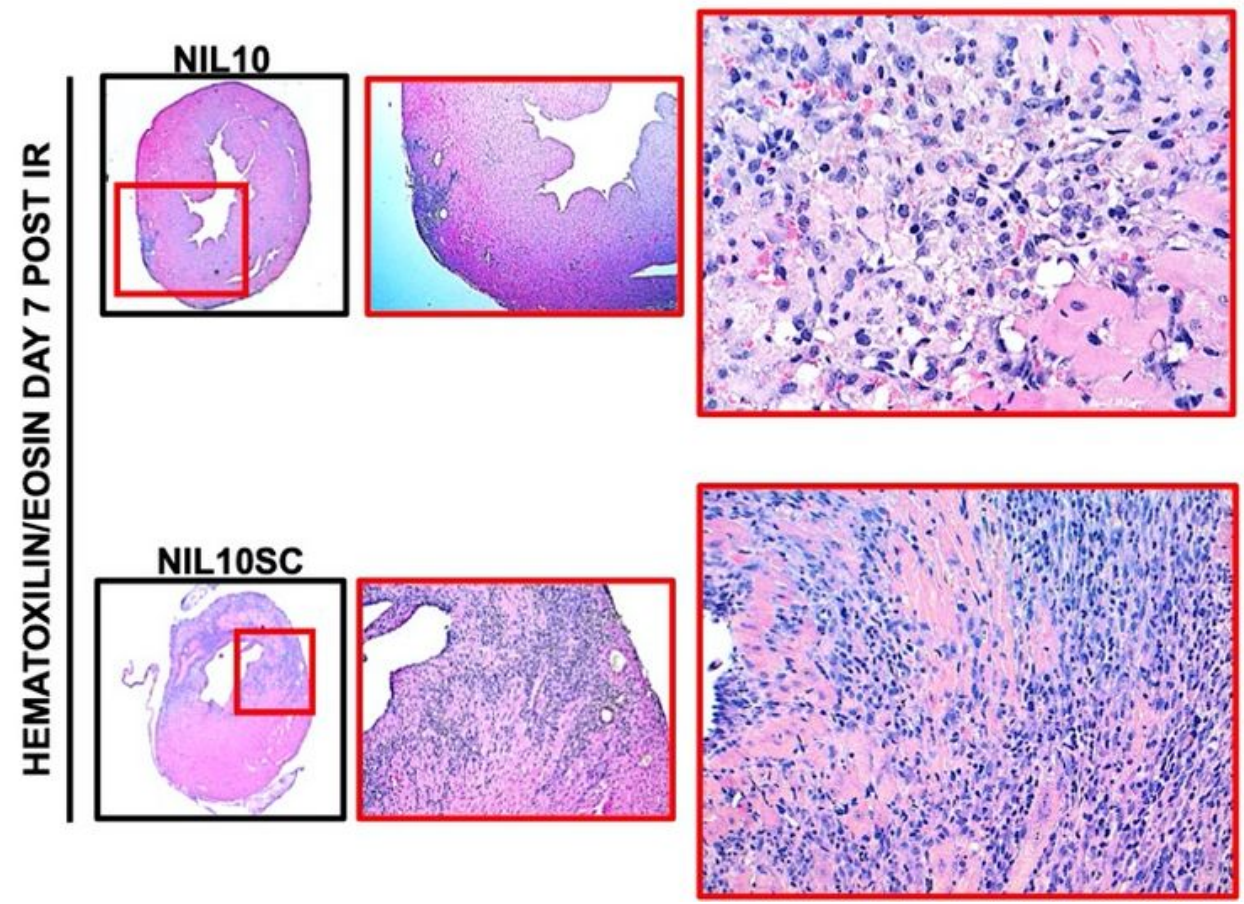

Figure 4 
Hematoxylin and Eosin staining of mouse hearts. Heart sections of mice subjected to IR and injected with NIL10 or NIL10SC, and collected by days 3 (A) or 7 (B) after IR. N=10 mice/group. From left to right: $2 X$, 20X, 40X.

\section{Figure 5}

A

\section{HN HN HN HN HN HN}

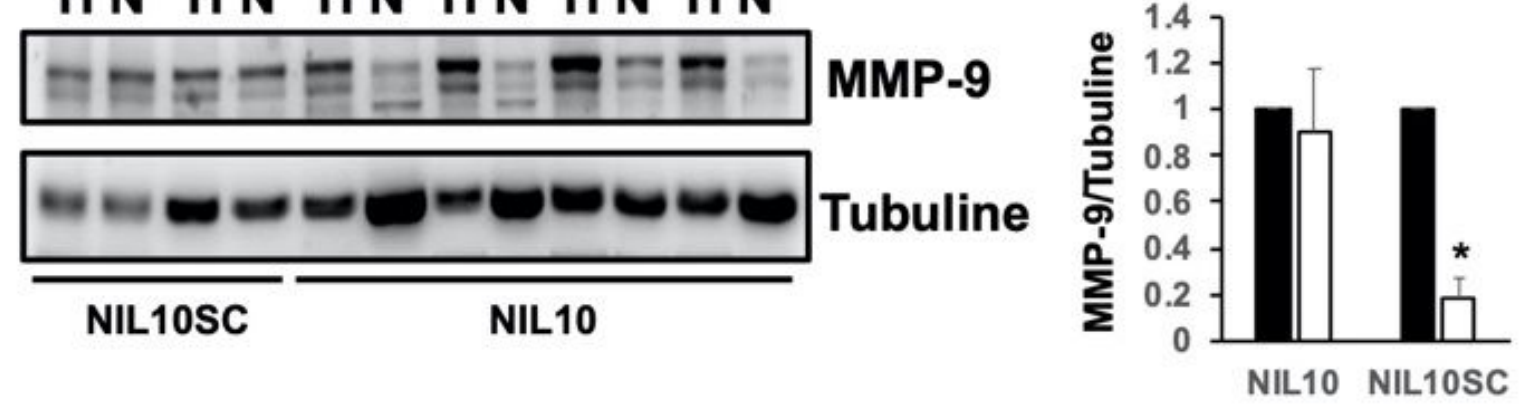

B
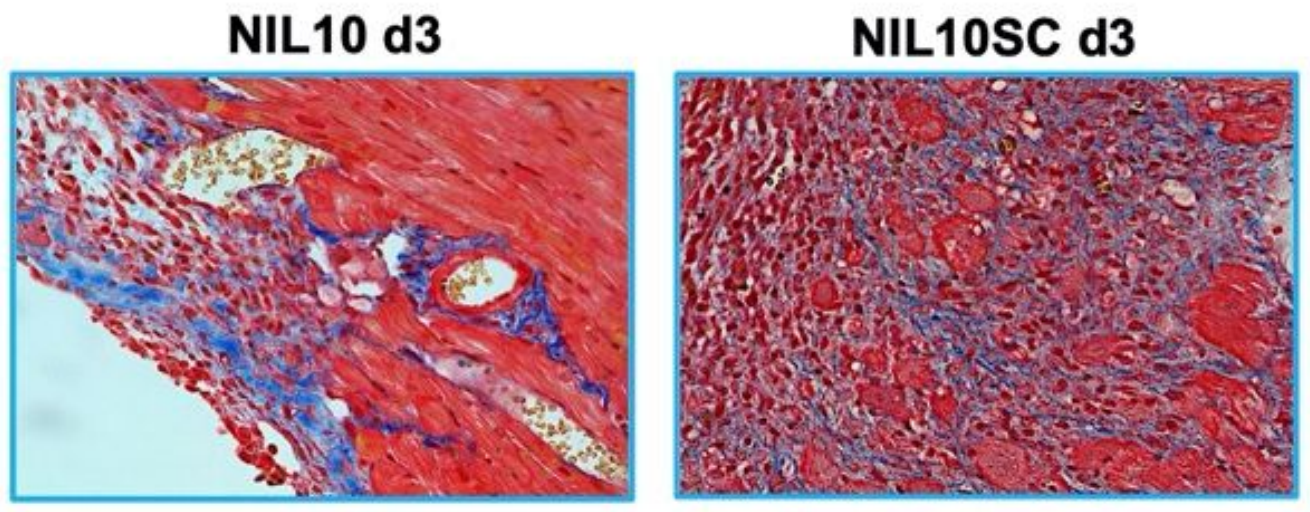

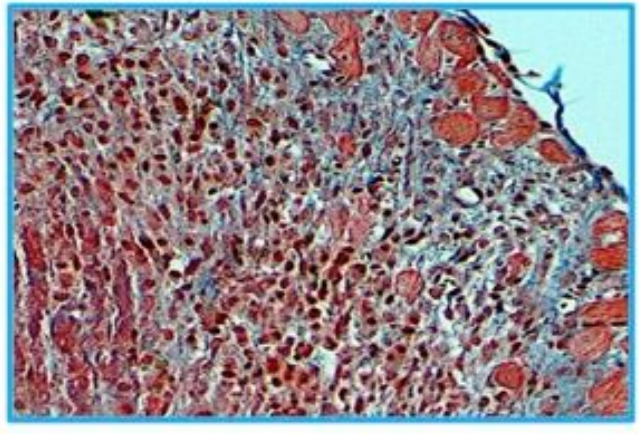

NIL10 d7

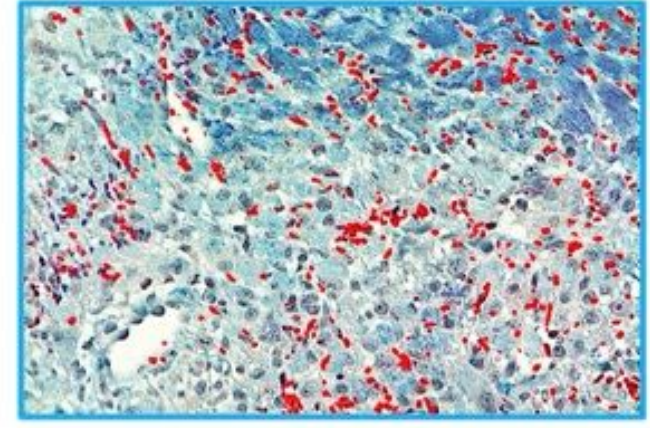

NIL10SC d7

Figure 5 
NIL10 reduce heart fibrosis in response to IR. A. Immunoblot detection of MMP9 from healthy $(\mathrm{H})$ or necrotic $(\mathrm{N})$ areas of mouse hearts after 7 days of IR and injected with NIL10 or NIL10SC. N=10 mice/group. Mean \pm SD. ${ }^{*} P<0.001$ NIL10 vs NIL10SC necrotic areas. B. Masson Trichrome staining of heart sections from mice injected with NIL10 or NIL10SC, after 3 or 7 days post IR ( $\mathrm{N}=10$ mice/group).

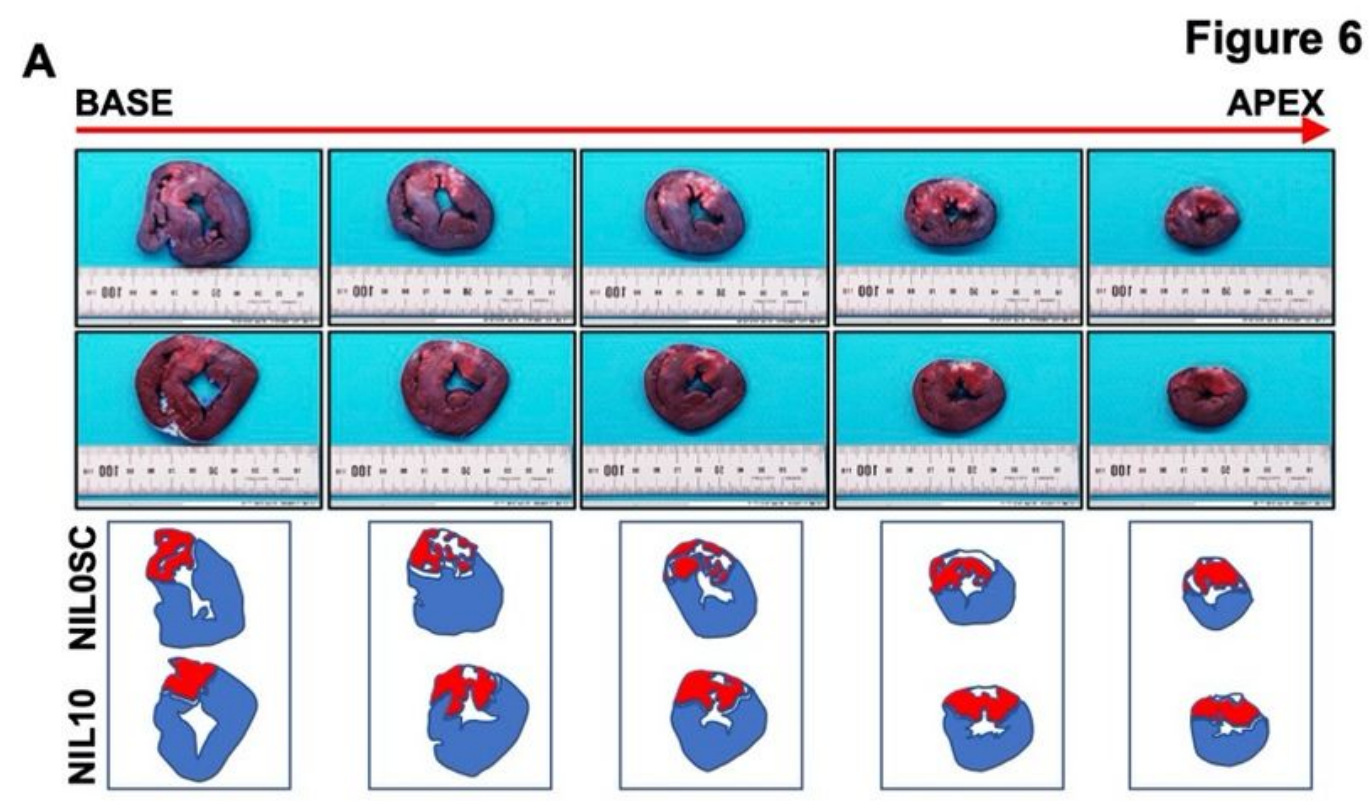

B

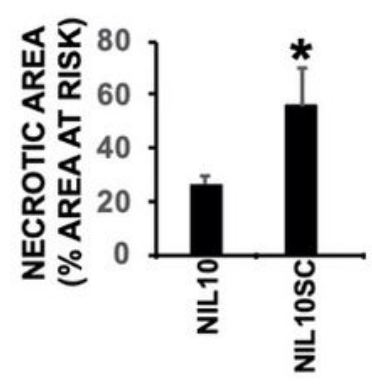

C

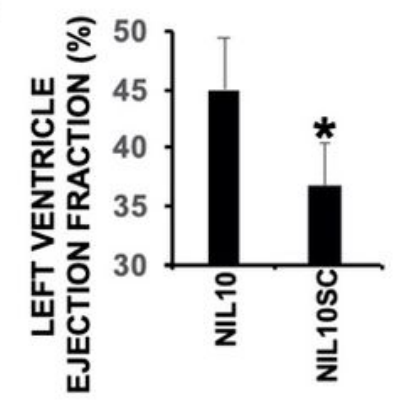

D

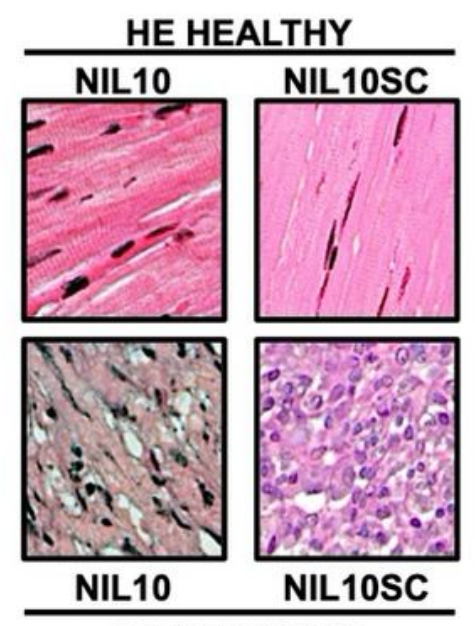

HE NECROTIC
E

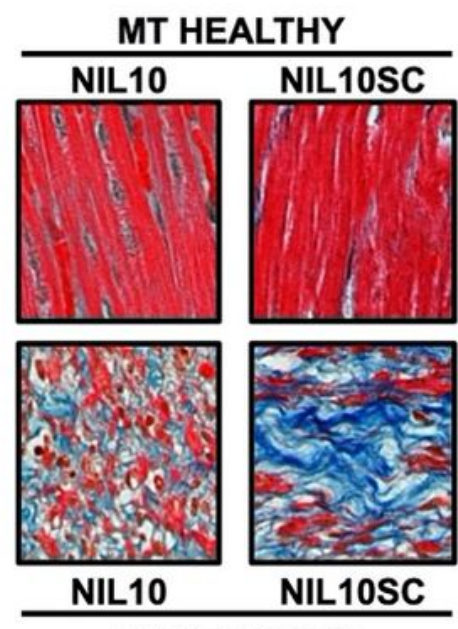

MT NECROTIC 


\section{Figure 6}

NIL10 induces cardiac protection in pigs subjected to IR. A. Evans blue/TTC staining (see methods for details) of pig heart sections (from apex to base) after 7 days of IR, showing healthy tissue (blue), the area at risk (red) and the necrotic areas (white). B. Measurement of necrotic areas of the hearts, represented as a percentage respect to the area at risk (Mean \pm SD. ${ }^{*} \mathrm{P}<0.001 \mathrm{NIL} 10$ vs NIL10SC, at day 7 post IR). C. Left ventricle ejection fraction of hearts from pigs injected with NIL10 or NIL10SC after 7 days of IR (Mean $\pm S D$. *P<0.001 NIL10 vs NIL10SC). D. Hematoxylin and Eosin staining of healthy and necrotic heart sections from pigs injected with NIL10 or NIL10SC after 7 days of IR. E. Masson Trichrome staining of the same hearts as in D. ( $\mathrm{N}=10$ pigs/group). 
DAY 3

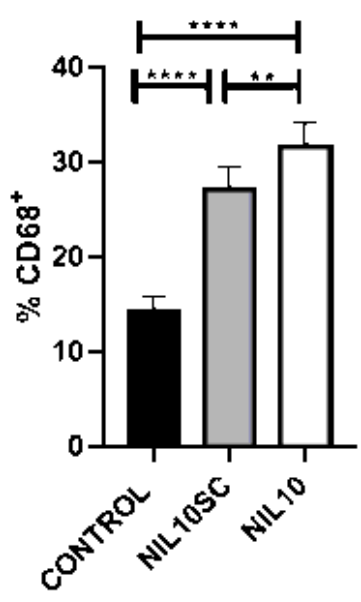

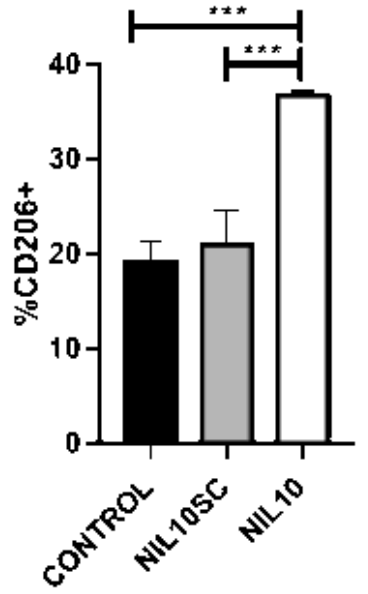

\section{DAY 7}
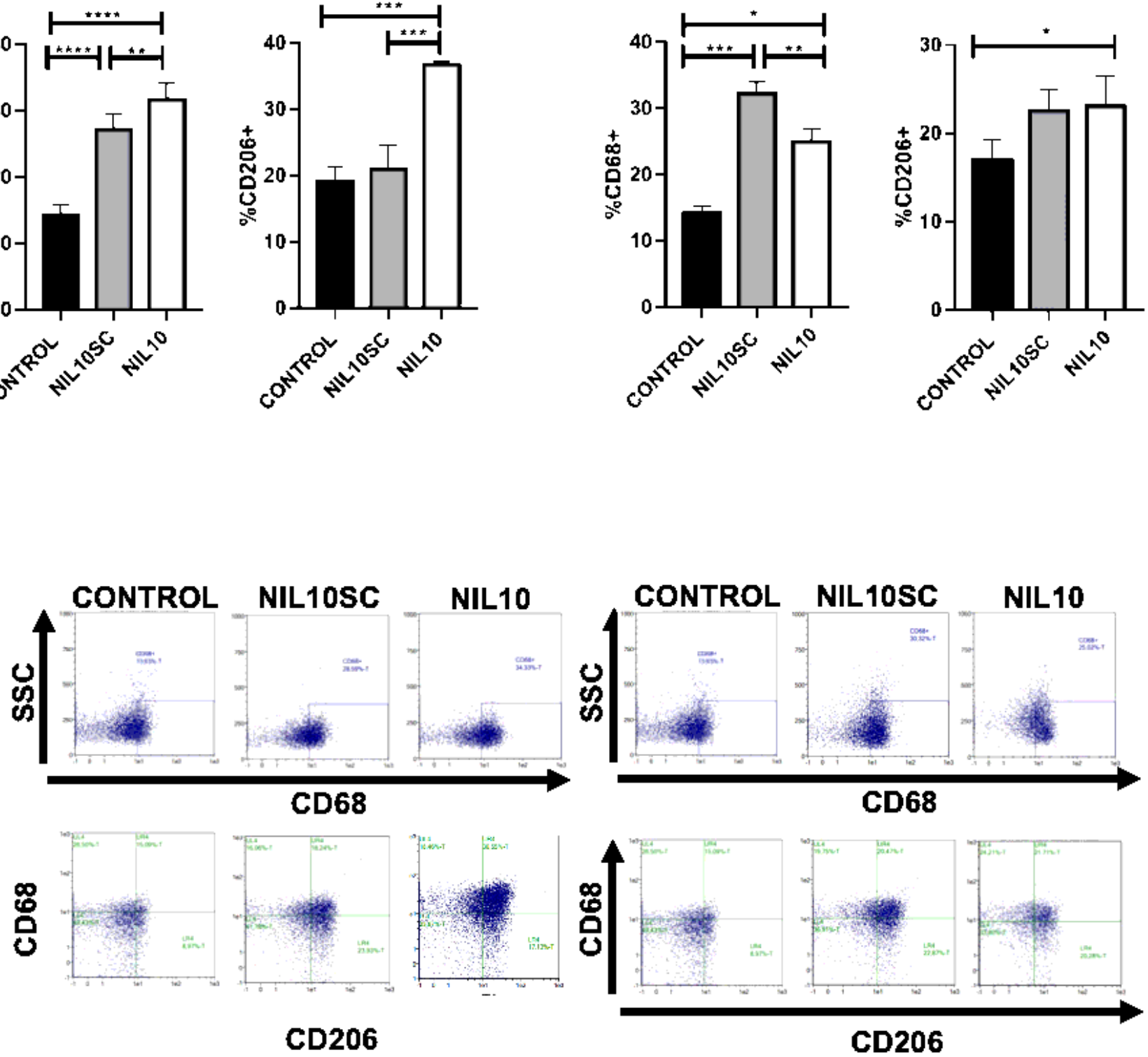

CD206

Figure 7

NIL10 induces M2 macrophage polarization in the hearts of mice subjected to IR. A. Flow cytometry analysis of macrophage populations in mouse hearts after 3 and 7 days of IR. The percentage of M2 CD68+/CD206+ population were selected from the necrotic and at risk areas of the hearts. B. Gating strategy used to identify macrophage-cell subsets in the healty and infarted mouse heart. A sequential gatting strategy was first used to identify M2 population expressing specific macrophage marker CD68, followed by the identification of the population with overlapping expression patterns (CD68/CD206) $(\mathrm{N}=10$ mice/group). Differences between groups were compared using one-way ANOVA. $* * P<0.01$. *** $\mathrm{P}<0.001 . * \star \star * \mathrm{P}<0.0001$. 


\begin{tabular}{c|c|c|c|}
\hline A Inflammatory & Anti-inflammatory & Chemokines \\
\cline { 2 - 4 } & C5a & IL1-RA & CXCL1 \\
G-CSF & IL-4 & CXCL2 \\
GM-CSF & IL-5 & CXCL3 \\
CCL1 & IL-7 & CXCL.9 \\
CCL11 & IL-10 & CXCL10 \\
IICAM-1 & IL-11 & CXCL11 \\
IFN-gamma & IL-13 & CXCL12 \\
IL-alpha & IL-16 & CCL2 \\
IL1-beta & IL27 & CCL3 \\
IL-2 & & CCL4 \\
IL-3 & & CCL5 (RANTES) \\
IL-6 & & CCL12 \\
IL12-P70 & & CCL17 \\
IL-17 & & \\
IL-23 & & \\
TNF-ALPHA & & \\
TREM-1 & & \\
MCSF & & \\
\hline
\end{tabular}

Figure 8

B

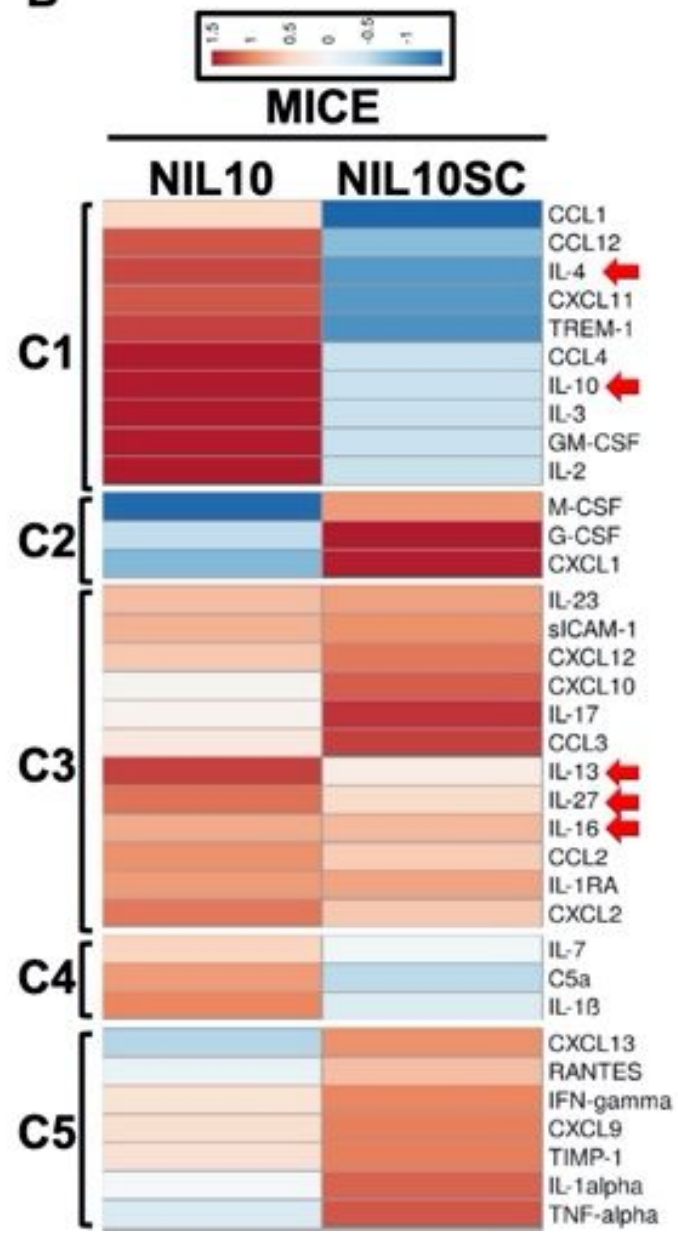

C

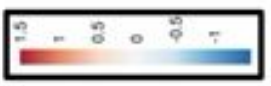

\section{PIGS}

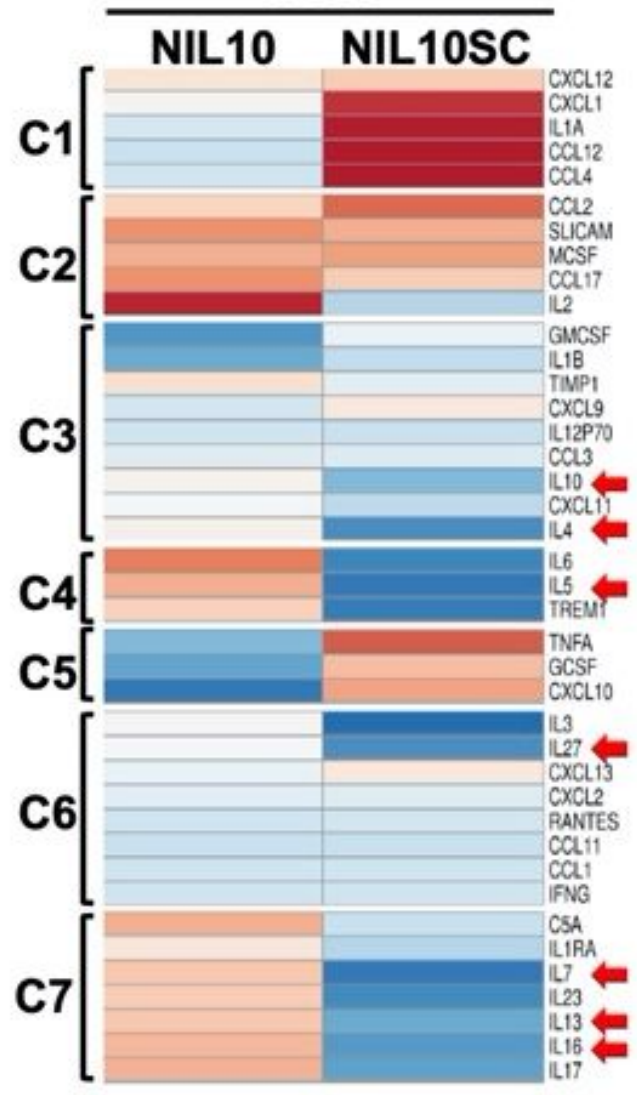

Figure 8

NIL10 induces the expression of resolving cytokines. A. Cytokine array used in the study. B. Clustered heat map of the differentially expressed cytokines in mice. C. Clustered heat map of the differentially expressed cytokines in pigs. Red arrows indicated anti-inflammatory cytokines. 
Figure 9

A
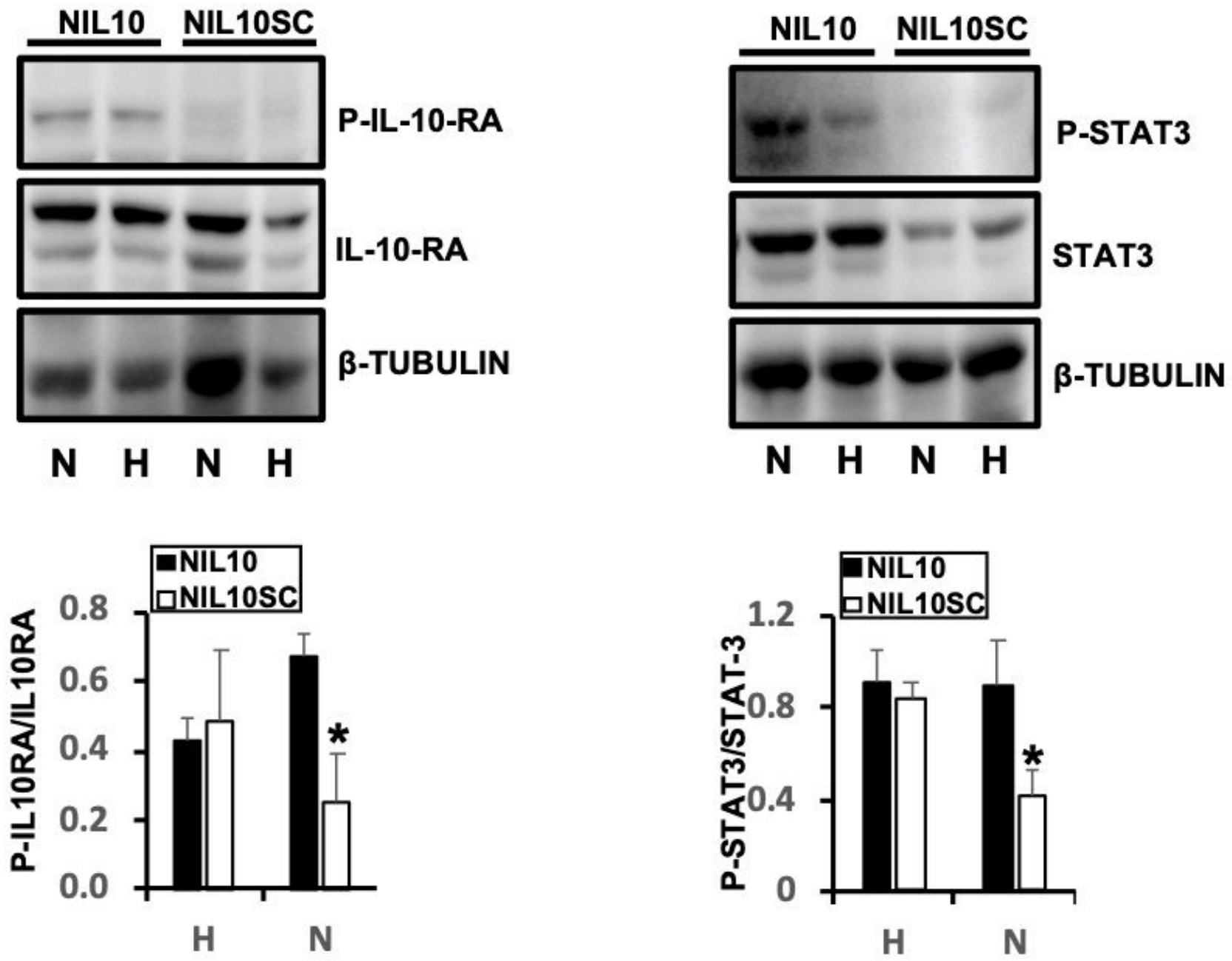

Figure 9

NIL10 induces the expression of resolving cytokines. A. Cytokine array used in the study. B. Clustered heat map of the differentially expressed cytokines in mice. C. Clustered heat map of the differentially expressed cytokines in pigs. Red arrows indicated anti-inflammatory cytokines. 
Figure 10

A

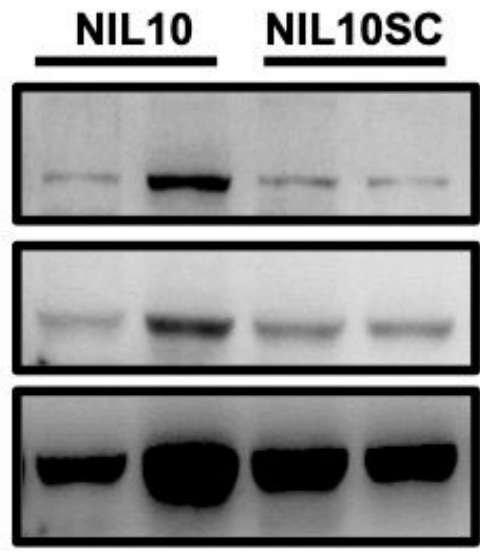

$\begin{array}{llll}\mathbf{H} & \mathbf{N} & \mathbf{H} & \mathbf{N}\end{array}$

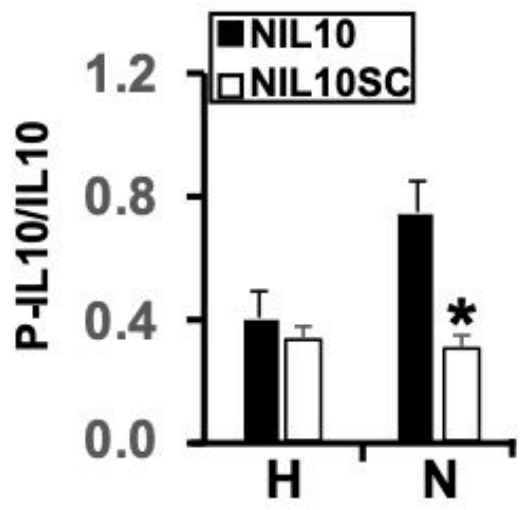

P-IL10R

IL10

\section{$\beta$-TUBULIN}
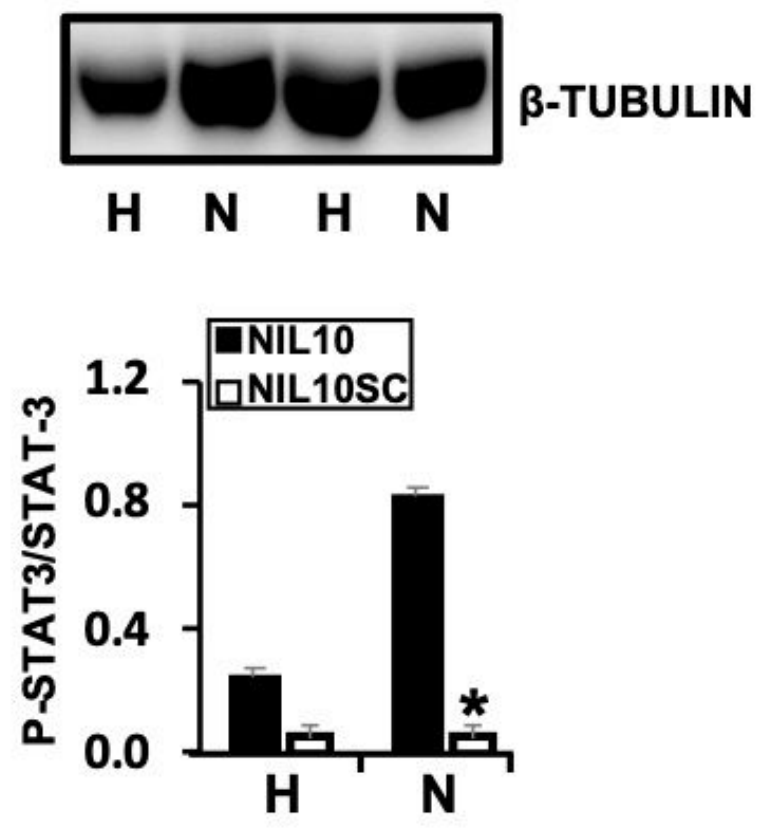

Figure 10

NIL10 induces phosphorylation of IL10RA and STAT3. A. Immunoblot detection of P-IL10-RA (upper), and total IL10-RA in the healthy $(\mathrm{H})$ and necrotic $(\mathrm{N})$ areas of pig hearts at day 7 after IR ( $<<0.05$ NIL10 vs NIL10SC necrotic areas). B. Immunoblot detection of P-STAT3, and total STAT3 in the same protein extracts. $\mathrm{N}=10$ pigs/group. Results expressed as Mean \pm SD ( $<<0.005$ NIL10 vs NIL10SC necrotic areas). 
A

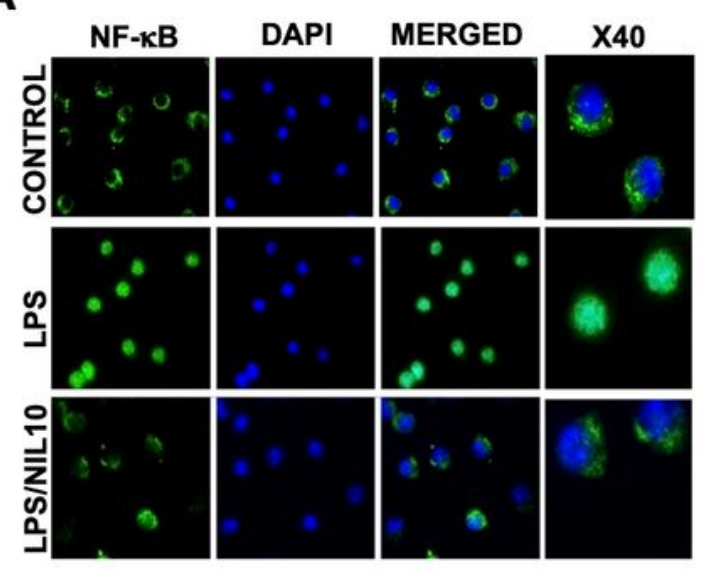

B Figure 11
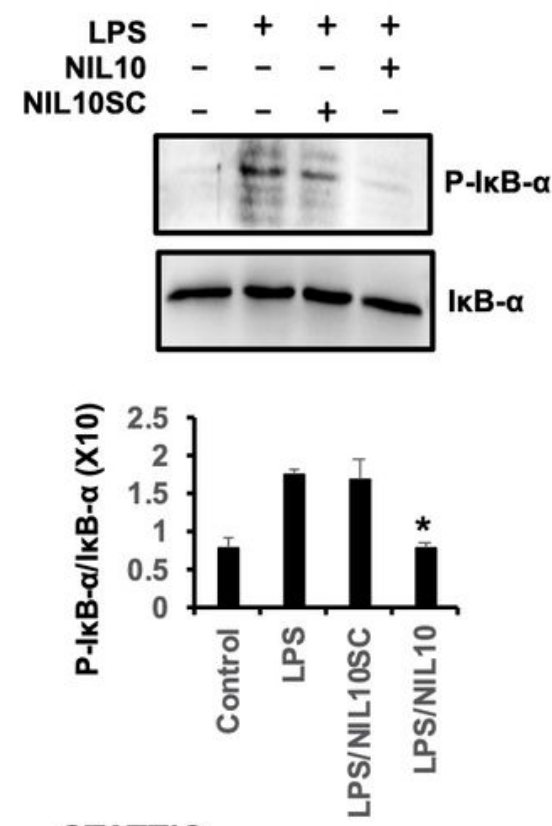

STATTIC
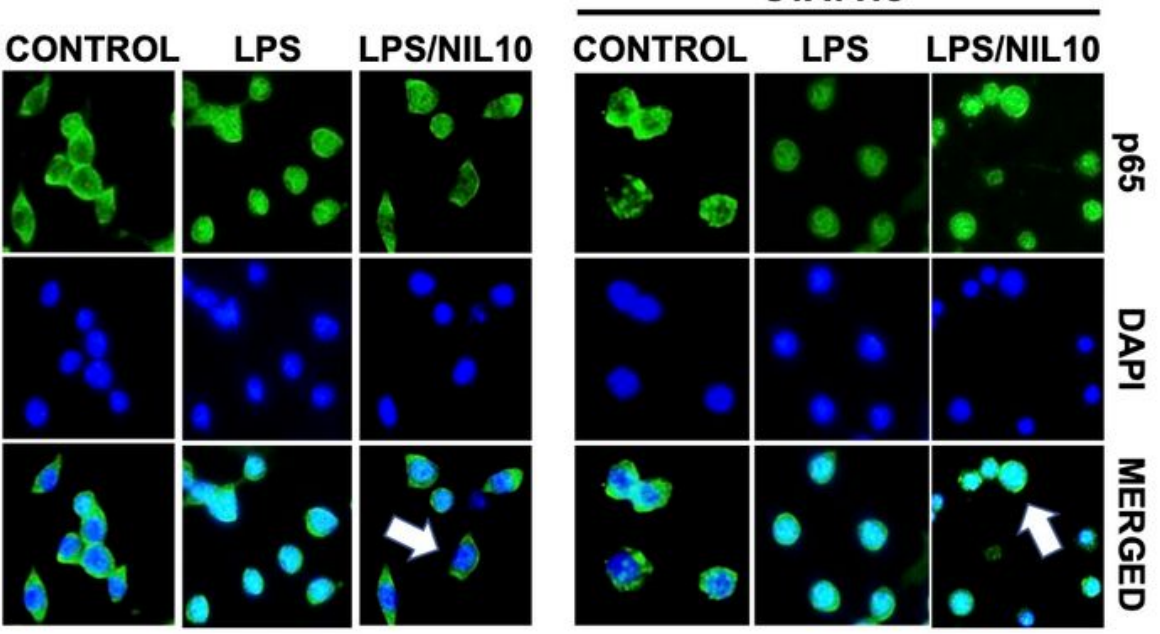

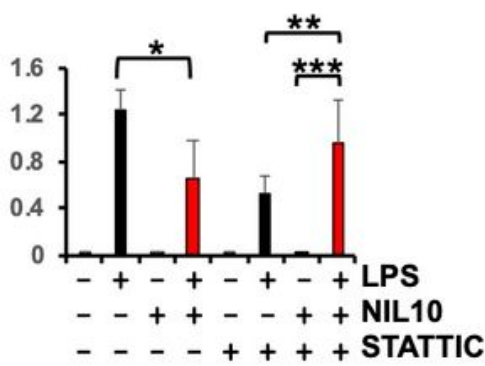

Figure 11

NIL10 prevents nuclear translocation of NF-KB trough STAT3 activation in RAW 264.7 macrophages. A. Confocal microscopy detection of NF- kB (p65) in RAW 264.7 cells stimulated with 500 mM LPS or in combination with NIL10. Nuclei were stained with DAPI. B. Immunoblot detection of P-IkB-a, or total IkB-a in RAW 264.7 cells treated with NIL10 or NIL10SC. (N=3, results expressed as Mean $\pm S D$. ${ }^{*}<0.05$ LPS/NIL10 vs LPS/NIL10SC). C. Confocal microscopy detection as in A, in which STATTIC, a 
pharmacological inhibitor of STAT3 was incubated. Arrows point nuclear or cytoplasmic localization of p65. Lower panels: immunoblot detection of iNOS in cells as in $\mathrm{C}$. $(\mathrm{N}=3$, results expressed as Mean $\pm \mathrm{SD}$. ${ }^{*} \mathrm{P}<0.03$ LPS vs LPS/NIL10 **P< 0.05 LPS/STATTIC vs LPS/NIL10SC/STATTIC. ***P<0.001 NIL10/STATIC vs LPS/NIL10/STATIC).

\section{Supplementary Files}

This is a list of supplementary files associated with this preprint. Click to download.

- TesoroetalJNB1GA.jpg 